\title{
A cohomological characterisation of Yu's property A for metric spaces
}

\author{
JACEK BRODZKI \\ GRAHAM A NiBLO \\ NICK WRIGHT
}

\begin{abstract}
We develop a new framework for cohomology of discrete metric spaces and groups which simultaneously generalises group cohomology, Roe's coarse cohomology, Gersten's $\ell^{\infty}$-cohomology and Johnson's bounded cohomology. In this framework we give an answer to Higson's question concerning the existence of a cohomological characterisation of Yu's property A, analogous to Johnson's characterisation of amenability. In particular, we introduce an analogue of invariant mean for metric spaces with property A. As an application we extend Guentner's result that box spaces of a finitely generated group have property A if and only if the group is amenable. This provides an alternative proof of Nowak's result that the infinite dimensional cube does not have property A.
\end{abstract}

55N91; 20J06, 30L05

There have been a number of cohomology theories introduced to study large scale geometry of groups and metric spaces, and important examples include: Gersten's $\ell^{\infty}$-cohomology which characterises hyperbolicity for a finitely presented group [5]; Johnson's bounded cohomology which characterises amenability for a locally compact group [7]; Roe's coarse cohomology introduced in [12] to define higher indices for elliptic operators on open manifolds. In this paper we introduce a new framework for cohomology of metric spaces and groups which generalises all of these examples and in addition allows us to define a new cohomology which detects Yu's property A, thereby answering a question of Higson.

The key ingredient which allows us to generalise from group cohomology to a theory for a metric space $X$ is a controlled support condition. Our coefficients $\mathcal{V}$ will therefore be modules equipped with a support function taking values in the power set of $X$. Classical examples arise from function spaces on $X$ equipped with the usual notion of support. The cohomology theories then arise from the bicomplex $\mathcal{E}^{p, q}(X, \mathcal{V})$ of controlled support cochains: 
- Given a metric space $X$ choose a base point $x \in X$ and let $\mathcal{R}_{x}$ be the vector space $\mathbb{R} \delta_{x}$ spanned by the delta function $\delta_{x} \in \ell^{1}(X)$. The cohomology $H_{\mathcal{E}}\left(X, \mathcal{R}_{x}\right)$ is Roe's coarse cohomology of $X$ with coefficients in $\mathbb{R}$.

- If $X$ is the underlying metric space of a discrete group $G$ of type $\mathcal{F}_{n}$, then the cohomology $H_{W}\left(X, \mathcal{R}_{e}\right)$ arising from a suitable completion of the bicomplex is Gersten's $\ell^{\infty}$-cohomology of $G$ with coefficients in $\mathbb{R}$. Hence Gersten's theory can be regarded as a completion of Roe's coarse cohomology and in this way can be extended to the context of metric spaces.

- Alternatively Gersten's cohomology is equivalent to the uncompleted cohomology $H_{\mathcal{E}}\left(X, \mathbb{R}_{\varnothing}\right)$ where every element of $\mathbb{R}$ is taken to have empty support.

- If $G$ is a group and the coefficients $V$ are equipped with a group action we may also consider the subcomplex of $G$-equivariant cochains. Setting $V_{c}$ to be the compactly supported elements of $V, H_{\mathcal{E}}(G, \mathcal{V})$ is the group cohomology of $G$ with coefficients in $V_{c}$. Furthermore the cohomology of the completed bicomplex agrees with the group cohomology of $G$ after completing the coefficients $V_{c}$.

- Now let $G$ be a group, $V$ be a $G$-Banach space and equip $V$ with the empty support function. Since every element of $V$ has compact supports the group cohomology $H^{*}(G, V)$ is equal to the equivariant bicomplex cohomology. Suitably augmenting the bicomplex we obtain a new chain complex whose cohomology agrees with Johnson's bounded cohomology $H_{b}^{*}(G, V)$.

- In general if $G$ is a group then the non-equivariant cohomology of the underlying metric space can be identified with the equivariant cohomology of $G$ by suitably twisting the coefficients. Hence as above it may be identified with the classical group cohomology.

- Now let $X$ be a metric space. The cochain $\mathcal{J}\left(x_{0}, x_{1}\right)=\delta_{x_{1}}-\delta_{x_{0}}$ defines a class in the cohomology $H_{W}^{1}\left(X, \ell_{0}^{1}\right)$ of the completed bicomplex which is trivial if and only if the space $X$ satisfies Yu's property A. Moreover, augmenting the bicomplex as before and completing we then obtain the complex of asymptotically invariant cochains and property $\mathrm{A}$ is characterised by the vanishing of asymptotically invariant cohomology.

We note in passing that combining the last two remarks one can characterise property A for a group in terms of group cohomology. This is related to the results in the articles [1; 2] by the authors and Nowak, and the article [4] by Douglas and Nowak.

The main focus of this paper is a detailed study of the results summarised in the last point of this list. We note that this is directly analogous to Johnson's characterisation 
of amenability in terms of bounded cohomology and take a moment to review that context.

A discrete group $G$ is said to be amenable if it has an invariant mean (see von Neumann [14]), that is, there exists an element $\mu \in\left(\ell^{\infty}(G)\right)^{*}=\left(\ell^{1}(G)\right)^{* *}$ such that $\mu(1)=1$ and $g \mu=\mu$ for all $g \in G$. Johnson's celebrated theorem [7] characterises amenability of a group $G$ in terms of vanishing of a particular cohomology class $[\mathcal{J}]$ in $H_{b}^{1}\left(G,\left(\ell_{0}^{1}(G)\right)^{* *}\right)$. As remarked in the our article [3], the function $\mathcal{J}$ gives rise to a class in classical group cohomology $H^{1}\left(G,\left(\ell_{0}^{1}(G)\right)^{* *}\right)$ and vanishing of this class also characterises amenability of the group. Indeed, given a cochain $\phi$ whose coboundary is $\mathcal{J}$, the difference $\delta-\phi$ is the required invariant mean, where $\delta$ denotes the Dirac function.

In geometric group theory, amenability is more often exhibited by the existence of a Følner or Reiter sequence (see Reiter [11]). The quotient completion $V_{Q}$ of a topological vector space $V$, introduced in this paper, allows us to interpret the Reiter sequence cohomologically. Specifically, the function $\mathcal{J}$ gives rise to a class in $H^{1}\left(G,\left(\ell_{0}^{1}(G)\right)_{Q}\right)$ and given a cochain $\phi$ with coboundary $\mathcal{J}$, the difference $\delta-\phi$ gives rise to a Reiter sequence. Regarding $\left(\ell_{0}^{1}(G)\right)^{* *}$ as the weak-* completion of $\ell_{0}^{1}(G)$ we see that both the invariant mean and the Reiter sequence arise in cohomology via a completion process.

In this paper, we exploit this idea to study property A which was introduced by Yu in his work on the coarse Baum-Connes conjecture [15] as a generalisation of the Følner condition for amenability to the context of a metric space. This property has become a focus for study in the crossover between non-commutative geometry and geometric group theory.

We will show that the following are equivalent:

- $X$ has property A;

- $X$ admits an asymptotically invariant mean (Theorem 7.3 part (5));

- The Johnson class of $X$ is trivial in controlled cohomology (Theorem 7.3 parts (2) and (3));

- The asymptotically invariant cohomology of $X$ vanishes (Theorem 9.6).

The reader will no doubt recognise that these results are parallel to Johnson's characterisation of amenability for a locally compact group [7]. As an application we provide a cohomological argument extending Guentner's result that box spaces of a finitely generated group have property A if and only if the group is amenable, (see Roe [13, 
Proposition 11.39]). This provides an alternative proof of Nowak's result that the union of finite cubes of all dimensions does not have property A.

The paper is organised as follows. In Section 1, by way of motivating what follows, we recall the definition of classical group cohomology and bounded cohomology and derive the forgetful map from bounded cohomology to classical cohomology via a bicomplex encoding them both. The rows of this bicomplex are acyclic and the forgetful map arises naturally from the collapse of the bicomplex onto the left hand column obtained by taking the augmentations. This point of view is reflected in Section 2 where we construct an algebraic bicomplex which will lead, via completion processes described in Section 3, to the definition of our controlled cohomology theories in Section 4. In Section 5 we pause to consider the case of the equivariant cohomology of a discrete group and show that the completions in this case may be carried out at the level of the coefficient module, making a link with group cohomology, and, via our results in [3], with the theory of Johnson and Ringrose. In Section 6 we introduce the notion of an asymptotically invariant mean. Asymptotic invariance is a cocycle condition, while we formulate the normalisation condition in terms of a map on coefficients. This leads naturally to consideration of the long exact sequence in cohomology arising from a short exact sequence of coefficient modules. In Section 7 we recall the definition of property A and give our first cohomological characterisation of property A in terms of vanishing of the Johnson class in controlled cohomology. We also show that this is equivalent to the existence of an asymptotically invariant mean on the space. In Section 8 we introduce asymptotically invariant cohomology and in Section 9 we show that vanishing of asymptotically invariant cohomology in dimensions greater than or equal to 1 is equivalent to property A. In Section 10 we consider two of the three known classes of spaces which do not have property A: box spaces of non-amenable residually finite groups, and the union of finite cubes of all dimensions. We use our cohomological approach to give a new unified proof that these classes do not satisfy property A. The remaining known example of a non-A space, that of an expander sequence, is considered from a cohomological point of view in a companion note (see the article [8] by the third author and Khukro).

Several of the results in this paper were announced at the Ascona conference in June 2009 , and the first and second authors would like to thank the organisers for their hospitality. This work is related in spirit to the results appearing in [4], in which Douglas and Nowak prove that exactness of a finitely generated group $G$ (which by work of Ozawa [10] is equivalent to property A) implies vanishing of the bounded cohomology $H_{b}^{q}(G, V)$, for so-called Hopf $G$-modules of continuous linear operators with values in $\ell^{\infty}(G)$. In [1;2] the results of Douglas and Nowak [4] are generalised 
to give a characterisation of topological amenability for a group action in terms of vanishing bounded cohomology.

This research was supported by EPSRC grant EP/F031947/1. We would like to thank Nigel Higson for asking the original question motivating these results and for his comments on an early draft of the paper. We would also like to thank Erik Guentner and Piotr Nowak for many stimulating discussions.

\section{Group cohomology}

In this section we will motivate the definitions to follow by examining the familiar objects of real valued group cohomology and bounded cohomology in a framework that will generalise to our context. In this section the group $G$ is taken to be a countable discrete group equipped with a proper left-invariant metric.

Recall that for a group $G$ the homogeneous bar resolution with real coefficients is given by the cochain complex $\left(C^{p}(G, \mathbb{R}), D\right)$ where $C^{p}(G, \mathbb{R})$ is the vector space of all $G$-invariant real valued functions on $G^{p+1}$, and $D$ is induced by the differential $\left(g_{0}, \ldots, g_{p}\right) \mapsto \sum_{i=0}^{p}(-1)^{p} \widehat{\mathbf{g}}_{i}$ (where $\widehat{\mathbf{g}_{i}}$ denotes $\left(g_{0}, \ldots, g_{p}\right)$ with the $i$ th term deleted). We regard $\mathbb{R}$ as a $G$-module with the trivial $G$-action, so that invariance of a function $\phi$ is equivalent to equivariance. The cohomology of this complex is the classical group cohomology $H^{p}(G, \mathbb{R})$.

On the other hand the bounded cohomology $H_{b}^{p}(G, \mathbb{R})$ is computed using the subcomplex of $\left(C^{p}(G, \mathbb{R}), D\right)$ consisting of bounded functions. The forgetful map which regards a bounded function as a function gives a map from $H_{b}^{p}(G, \mathbb{R})$ to $H^{p}(G, \mathbb{R})$.

We give an alternative description of this setup using the following bicomplex. Let $\mathcal{E}^{p, q}(G, \mathbb{R})$ consist of those real valued $G$-equivariant functions on $G^{p+1} \times G^{q+1}$, that are bounded over $\left\{\left(\left(g_{0}, \ldots, g_{p}\right),\left(h_{0}, \ldots, h_{q}\right)\right) \mid d\left(g_{i}, g_{j}\right) \leq R\right.$ for all $\left.i, j\right\}$ for each $R$. There are natural anti-commuting differentials

$$
D: \mathcal{E}^{p, q}(G, \mathbb{R}) \rightarrow \mathcal{E}^{p+1, q}(G, \mathbb{R}), d: \mathcal{E}^{p, q}(G, \mathbb{R}) \rightarrow \mathcal{E}^{p, q+1}(G, \mathbb{R})
$$

induced by the standard differential as above, so we may construct the totalised complex and compute its cohomology.

It is easy to see that the rows of the bicomplex (which correspond to fixing $p$ and varying $q$ ) are exact. A splitting is given by setting

$$
s \phi\left(\left(g_{0}, \ldots, g_{p}\right),\left(h_{0}, \ldots, h_{q}\right)\right)=\phi\left(\left(g_{0}, \ldots, g_{p}\right),\left(g_{0}, h_{0}, \ldots, h_{q}\right)\right) .
$$

Standard arguments then show that the cohomology of the bicomplex collapses onto the left hand column obtained by augmenting the rows. This means that taking $\mathcal{E}^{p,-1}(G, \mathbb{R})$ 
to be equal to the kernel of the differential $d: \mathcal{E}^{p, 0}(G, \mathbb{R}) \rightarrow \mathcal{E}^{p, 1}(G, \mathbb{R})$, we obtain a cochain complex $\left(\mathcal{E}^{p,-1}(G, \mathbb{R}), D\right)$ and that the cohomology of this complex coincides with the cohomology of the totalised complex.

The cocycles in $\left(\mathcal{E}^{p,-1}(G, \mathbb{R}), D\right)$ are equivariant functions $\phi: G^{p+1} \times G \rightarrow \mathbb{R}$ which are constant in the final variable. The constraint that these functions should be bounded over the subset $\left\{\left(\left(g_{0}, \ldots, g_{p}\right),\left(h_{0}\right)\right) \mid d\left(g_{i}, g_{j}\right) \leq R\right.$ for all $\left.i, j\right\}$ is then trivially satisfied by equivariance, since properness of the metric ensures that there are only finitely many orbits of points $\left(g_{0}, \ldots, g_{p}\right)$ for which $d\left(g_{i}, g_{j}\right) \leq R$ for all $i, j$. Hence the cochain complex $\left(\mathcal{E}^{p,-1}(G, \mathbb{R}), D\right)$ is isomorphic to the cochain complex $\left(C^{p}(G, \mathbb{R}), D\right)$ and its cohomology is standard group cohomology.

There is a second augmentation that we may construct by taking the kernels $\mathcal{E}^{-1, q}(G, \mathbb{R})$ of the maps $D: \mathcal{E}^{0, q}(G, \mathbb{R}) \rightarrow \mathcal{E}^{1, q}(G, \mathbb{R})$. Since the columns are not acyclic the corresponding cochain complex will not compute the cohomology of the bicomplex. Instead we claim that it computes the bounded cohomology of the group. To see this note that elements of the kernel are constant in the first variable, and that the boundedness condition simplifies to the condition that the cocycles are bounded over all choices of $\left(h_{0}, \ldots, h_{q}\right)$. Hence the kernels give rise to a cochain complex isomorphic to the complex of bounded equivariant functions on the group, and the cohomology is the bounded cohomology of $G$.

Finally we consider the map on cohomology induced by the inclusion of $\left(\mathcal{E}^{-1, q}(G, \mathbb{R}), d\right)$ into the bicomplex. It is routine to establish that this is the standard forgetful map from bounded to ordinary cohomology.

It should be noted that this discussion applies more generally to group cohomology with coefficients in any Banach $G$-module.

In what follows we will generalise this construction to give cohomology theories which detect property A for a metric space. The principal ingredients are the definition of a module over a space and the construction of a bicomplex in which the notion of controlled support acts as a proxy for invariance under a group action. The cohomology of the completed bicomplex (the controlled cohomology of $X$ ) is analogous to group cohomology, while an augmentation of the vertical differential will provide an analogue of bounded cohomology, the asymptotically invariant cohomology of $X$. While the controlled cohomology detects property A it does not necessarily vanish for a property A space, any more than group cohomology necessarily vanishes for an amenable group. On the other hand the asymptotically invariant cohomology vanishes for a property A space, just as bounded cohomology vanishes for an amenable group. 


\section{The algebraic bicomplex}

In order to define a suitable cohomology theory we need first to define the notion of a module over a metric space. The definition is motivated by considering the case of the classical Banach $G$-module of bounded, equivariant, real valued functions on a group $G$. Each element of the module is equipped with a support in the group, consisting of those elements for which the function is non-zero. The equivariance condition controls the variation of the support of a function. In the absence of a group action we want to capture some notion of controlled supports, but we need to do so in a "coarse" manner:

Definition 2.1 Let $X$ be a topological space. An $X$-module consists of a triple $\mathcal{V}=(V,\|\cdot\|$, Supp $)$ where the pair $(V,\|\cdot\|)$ is a Banach space, and Supp is a function (the support function) from $V$ to the set of closed subsets of $X$ satisfying the following axioms:

(1) $\operatorname{Supp}(v)=\varnothing$ if $v=0$,

(2) $\operatorname{Supp}(v+w) \subseteq \operatorname{Supp}(v) \cup \operatorname{Supp}(w)$ for every $v, w \in V$,

(3) $\operatorname{Supp}(\lambda v)=\operatorname{Supp}(v)$ for every $v \in V$ and every $\lambda \neq 0$.

(4) if $v_{n}$ is a sequence converging to $v$ then $\operatorname{Supp}(v) \subseteq \overline{\bigcup_{n} \operatorname{Supp}\left(v_{n}\right)}$.

Example 2.2 Let $X$ be a topological space and let $V=\ell^{1}(X)$ be equipped with the $\ell^{1}$-norm. The standard support structure for this module sets

$$
\operatorname{Supp}(f)=\overline{\{x \in X \mid f(x) \neq 0\}}
$$

for each $f \in \ell^{1}(X)$.

Note that if $(W,\|\cdot\|)$ is a closed subspace of $(V,\|\cdot\|)$ then any support function on $X$ restricts to a support function $\left.\operatorname{Supp}\right|_{W}$ on $W$ so that $\left(W,\|\cdot\|,\left.\operatorname{Supp}\right|_{W}\right)$ is also an $X$-module. We will later consider the special case of the subspace $\ell_{0}^{1}(X)$ of $\ell^{1}(X)$ consisting of functions $f$ such that $\sum_{x \in X} f(x)=0$, by analogy with the Johnson-Ringrose characterisation of amenability.

If $X$ is equipped with a $G$ action for some group $G$, then we may also consider the notion of a $G$-equivariant $X$ module. This is an $X$-module $(V,\|\cdot\|$, Supp) equipped with a linear isometric action of $G$ such that $g \operatorname{Supp}(v)=\operatorname{Supp}(g v)$ for every $g \in G$ and every $v \in V$.

Let $X$ be a metric space, let $G$ be a group acting by isometries on $X$ and let $\mathcal{V}=\left(V,\|\cdot\|_{V}\right.$, Supp $)$ be a $G$-equivariant $X$ module. Associated to this data we 
will construct an algebraic bicomplex $\mathcal{E}^{p, q}(X, \mathcal{V})$. This bicomplex also depends on the group $G$, however for concision we will generally omit $G$ from our notation.

For $\mathbf{x} \in X^{p+1}, \mathbf{y} \in X^{q+1}$, we adopt the standard convention that coordinates of $\mathbf{x}, \mathbf{y}$ are written $x_{0}, \ldots, x_{p}$ and $y_{0}, \ldots, y_{q}$.

For a positive real number $R$ let

$$
\begin{aligned}
\Delta_{R}^{p+1} & :=\left\{\mathbf{x} \in X^{p+1} \mid d\left(x_{i}, x_{j}\right) \leq R, \forall i, j\right\} \\
\Delta_{R}^{p+1, q+1}: & =\left\{(\mathbf{x}, \mathbf{y}) \in X^{p+1} \times X^{q+1} \mid d(u, v) \leq R, \forall u, v \in\left\{x_{0}, \ldots x_{p}, y_{0}, \ldots, y_{q}\right\}\right\} .
\end{aligned}
$$

Identifying $X^{p+1} \times X^{q+1}$ with $X^{p+q+2}$ in the obvious way, $\Delta_{R}^{p+1, q+1}$ can be identified with $\Delta_{R}^{p+q+2}$. Given a function $\phi: X^{p+1} \times X^{q+1} \rightarrow V$ we set

$$
\|\phi\|_{R}=\sup _{\mathbf{x} \in \Delta_{R}^{p+1}, \mathbf{y} \in X^{q+1}}\|\phi(\mathbf{x}, \mathbf{y})\|_{V} .
$$

Definition 2.3 (i) We say that a function $\phi$ is of controlled supports if for every $R>0$ there exists $S>0$ such that whenever $(\mathbf{x}, \mathbf{y}) \in \Delta_{R}^{p+1, q+1}$ then $\operatorname{Supp}(\phi(\mathbf{x}, \mathbf{y}))$ is contained in $B_{S}(u)$ for all $u \in\left\{x_{0}, \ldots x_{p}, y_{0}, \ldots, y_{q}\right\}$.

(ii) We denote by $\mathcal{E}^{p, q}(X, \mathcal{V})$ the space of all $G$-equivariant maps $\phi: X^{p+1} \times$ $X^{q+1} \rightarrow V$ which are of controlled supports and such that $\|\phi\|_{R}<\infty$ for all $R>0$. Where the context is clear we will abbreviate $\mathcal{E}^{p, q}(X, \mathcal{V})$ to $\mathcal{E}^{p, q}$.

We equip the space $\mathcal{E}^{p, q}(X, \mathcal{V})$ with the topology arising from the semi-norms $\|\cdot\|_{R}$. While it is natural to allow $R$ to range over all positive values we note that the topology this induces is the same as the topology arising from the countable family of seminorms $\|\cdot\|_{R}$ for $R \in \mathbb{N}$.

The usual boundary map $\partial: X^{m+1} \mapsto X^{m}$ induces a pair of anti-commuting coboundary maps $D, d$ which yield the bicomplex

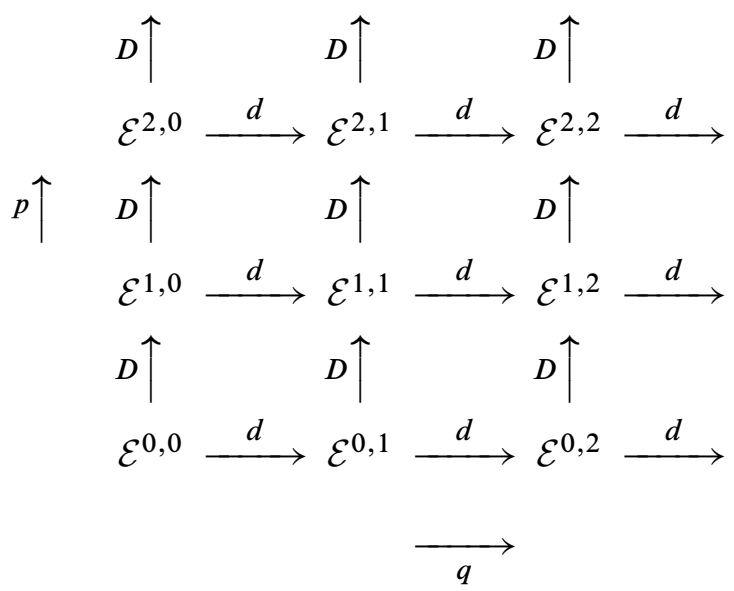


Specifically, $D: \mathcal{E}^{p, q} \rightarrow \mathcal{E}^{p+1, q}$ is given by

$$
D \phi\left(\left(x_{0}, \ldots, x_{p+1}\right), \mathbf{y}\right)=\sum_{i=0}^{p+1}(-1)^{i} \phi\left(\left(x_{0}, \ldots, \hat{x}_{i}, \ldots, x_{p+1}\right), \mathbf{y}\right)
$$

while $d: \mathcal{E}^{p, q} \rightarrow \mathcal{E}^{p, q+1}$ is

$$
d \phi\left(\mathbf{x},\left(y_{0}, \ldots, y_{q+1}\right)\right)=\sum_{i=0}^{q+1}(-1)^{i+p} \phi\left(\mathbf{x},\left(y_{0}, \ldots, \hat{y}_{i}, \ldots, y_{q+1}\right)\right) .
$$

We note that the definition of $\mathcal{E}^{p, q}$ and the maps $D: \mathcal{E}^{p, q} \rightarrow \mathcal{E}^{p+1, q}, d: \mathcal{E}^{p, q} \rightarrow \mathcal{E}^{p, q+1}$ make sense not just for positive $p, q$ but also when one of $p$ or $q$ is -1 . Elements of $\mathcal{E}^{p,-1}(X, \mathcal{V})$ are maps $\phi: X^{p+1} \times X^{0} \rightarrow V$; for convenience of notation, we will suppress the $X^{0}$ factor, and write $\phi(\mathbf{x})$ for $\phi(\mathbf{x},())$. We will identify $\mathcal{E}^{p,-1}(X, \mathcal{V})$ as the augmentation of row $p$, noting that the augmentation map is the differential $d: \mathcal{E}^{p,-1} \rightarrow \mathcal{E}^{p, 0}$ defined by $d \phi(\mathbf{x},(y))=\phi(\mathbf{x},(\hat{y}))$. Suppressing the empty vector we see that $d \phi(\mathbf{x},(y))=\phi(\mathbf{x})$, that is, $d$ is the inclusion of $\mathcal{E}^{p,-1}(X, \mathcal{V})$ into $\mathcal{E}^{p, 0}(X, \mathcal{V})$ as functions which are constant in the $y$ variable.

Lemma 2.4 The maps $D$ and $d$ are well-defined, continuous, anti-commuting differentials.

Proof The fact that $D$ and $d$ are anti-commuting differentials on the larger space of all equivariant functions from $X^{p+1} \times X^{q+1}$ to $V$ is standard. We must show that $D, d$ preserve finiteness of the semi-norms, and controlled supports. We note that $\|D \phi\|_{R} \leq(p+2)\|\phi\|_{R}$ by the triangle inequality, and a corresponding estimate holds for $\|d \phi\|_{R}$. Hence $D, d$ are continuous, and the semi-norms are finite as required.

For $\phi$ of controlled supports we now show that $D \phi$ is of controlled supports. Given $R>0$, take $(\mathbf{x}, \mathbf{y}) \in \Delta_{R}^{p+2, q+1}$. Since $\phi$ is of controlled supports, there exists $S$ such that the support $\operatorname{Supp}\left(\phi\left(\left(x_{0}, \ldots, \hat{x}_{i}, \ldots, x_{p+1}\right), \mathbf{y}\right)\right)$ is contained in $B_{S}\left(x_{i^{\prime}}\right)$ and $B_{S}\left(y_{j}\right)$ for all $i^{\prime} \neq i$, and for all $j$. Since for any $i^{\prime} \neq i$ we have $d\left(x_{i}, x_{i^{\prime}}\right) \leq R$ we deduce that $\operatorname{Supp}\left(\phi\left(\widehat{\mathbf{x}}_{i}, \mathbf{y}\right)\right)$ lies in $B_{S+R}\left(x_{i^{\prime}}\right)$ for all $i^{\prime}$. By the axioms for Supp the support of $D \phi$ is contained in $B_{S+R}\left(x_{i^{\prime}}\right)$ and $B_{S}\left(y_{j}\right)$ for all $i^{\prime}$ and all $j$, since this holds for the summands.

The argument for $d \phi$ is identical, exchanging the roles of $\mathbf{x}, \mathbf{y}$.

We note in passing that continuity of the differentials will enable us to extend them to differentials on completions of the algebraic bicomplex defined in Section 3. 
Definition 2.5 We denote by $H_{\mathcal{E}}^{*}(X, \mathcal{V})$ the cohomology of the totalisation of the bicomplex $\mathcal{E}^{p, q}, p, q \geq 0$, with the differentials $D, d$.

Remark If $X$ is equipped with two coarsely equivalent $G$-invariant metrics $d, d^{\prime}$ then for any module over $X$ the controlled support conditions arising from these metrics are the same. Moreover the family of semi-norms is equivalent in the sense that for each $R$ there is an $S$ such that $\|\cdot\|_{R, d} \leq\|\cdot\|_{S, d^{\prime}}$ and for each $R$ there is an $S$ such that $\|\cdot\|_{R, d^{\prime}} \leq\|\cdot\|_{S, d}$. Hence the bicomplexes and the cohomology we obtain from each metric are identical. This applies in particular if $X=G$ is a countable group and the two metrics are both left-invariant proper metrics on $G$.

We will now demonstrate exactness of the rows in the augmented complex. This allows the cohomology of the totalisation to be computed in terms of the left-hand column.

Proposition 2.6 For each $p$ the augmented row $\left(\mathcal{E}^{p, *}(X, \mathcal{V}), d\right), p \geq-1$ is exact. Specifically, for all $p \geq 0$ there is a continuous splitting $s: \mathcal{E}^{p, q} \rightarrow \mathcal{E}^{p, q-1}$ given by $s \phi\left(\left(x_{0}, \ldots, x_{p}\right),\left(y_{0}, \ldots, y_{q-1}\right)\right)=(-1)^{p} \phi\left(\left(x_{0}, \ldots, x_{p}\right),\left(x_{0}, y_{0}, \ldots, y_{q-1}\right)\right)$.

We have $(d s+s d) \phi=\phi$ for $\phi \in \mathcal{E}^{p, q}$ with $p \geq 0$, and $s d \phi=\phi$ for $\phi$ in $\mathcal{E}^{p,-1}$.

Proof The fact that $s$ defines a splitting on the larger space of all equivariant functions from $X^{p+1} \times X^{q+1}$ to $V$ is standard homological algebra. We must verify that if $\phi$ is of controlled supports then so is $s \phi$, and that if $\phi$ has bounded $R$-norms then so does $s \phi$. The latter will follow from continuity of $s$, which will also allow us to extend the splitting to the completed complexes later on.

Continuity is straightforward. For each $R \geq 0$ we have $\|s \phi\|_{R} \leq\|\phi\|_{R}$; this is immediate from the observation that if $\left(x_{0}, \ldots, x_{p}\right)$ in $\Delta_{R}^{p+1}$ then

$$
\left\|\phi\left(\left(x_{0}, \ldots, x_{p}\right),\left(x_{0}, y_{0}, \ldots, y_{q-1}\right)\right)\right\|_{V} \leq\|\phi\|_{R} .
$$

It remains to verify that $s \phi$ is of controlled supports. Given $R>0$, since $\phi$ is of controlled supports we know there exists $S$ such that if $(\mathbf{x}, \mathbf{y}) \in \Delta_{R}^{p+1, q+1}$ then the support $\operatorname{Supp}(\phi(\mathbf{x}, \mathbf{y}))$ is contained in $B_{S}\left(x_{i}\right)$ and $B_{S}\left(y_{j}\right)$ for all $i, j$. If the element $\left(\left(x_{0}, \ldots, x_{p}\right),\left(y_{0}, \ldots, y_{q-1}\right)\right)$ lies in $\Delta_{R}^{p+1, q}$ then $\left(\left(x_{0}, \ldots, x_{p}\right),\left(x_{0}, y_{0}, \ldots, y_{q-1}\right)\right)$ lies in $\Delta_{R}^{p+1, q+1}$, hence the support $\operatorname{Supp}\left(s \phi\left(\left(x_{0}, \ldots, x_{p}\right),\left(y_{0}, \ldots, y_{q-1}\right)\right)\right)$ is also contained in $B_{S}\left(x_{i}\right)$ and $B_{S}\left(y_{j}\right)$ for all $i, j$.

This completes the proof. 
We remark that the corresponding statement is false for the vertical differential $D$, since for $\phi \in \mathcal{E}^{p, q}(X, \mathcal{V})$, the function

$$
\left(\left(x_{0}, \ldots, x_{p-1}\right),\left(y_{0}, \ldots, y_{q}\right)\right) \mapsto \phi\left(\left(y_{0}, x_{0}, \ldots, x_{p-1}\right),\left(y_{0}, \ldots, y_{q}\right)\right.
$$

is only guaranteed to be bounded on sets of the form

$$
\left\{\left(\left(x_{0}, \ldots, x_{p-1}\right),\left(y_{0}, \ldots, y_{q}\right)\right) \mid d(u, v) \leq R \text { for all } u, v \in\left\{x_{0}, \ldots, x_{p-1}, y_{0}\right\}\right\},
$$

and not on $\Delta_{R}^{p} \times X^{q+1}$.

Corollary 2.7 The cohomology $H_{\mathcal{E}}^{*}(X, \mathcal{V})$ is isomorphic to the cohomology of the cochain complex $\left(\mathcal{E}^{*,-1}(X, \mathcal{V}), D\right)$.

Proof This follows from the exactness of the augmented rows of the bicomplex - the cocycle $\phi \in \mathcal{E}^{p, q}(X, \mathcal{V})$ is cohomologous to the cocycle $(-D s)^{q}(\phi) \mathcal{E}^{p+q, 0}(X, \mathcal{V})$, whence $H_{\mathcal{E}}^{*}(X, \mathcal{V})$ is isomorphic to the cohomology of the complex $\operatorname{ker}\left(d: \mathcal{E}^{p, 0} \rightarrow\right.$ $\left.\mathcal{E}^{p, 1}\right)$ with the differential $D$. The augmentation map $d: \mathcal{E}^{p,-1} \rightarrow \mathcal{E}^{p, 0}$ yields an isomorphism from $\left(\mathcal{E}^{p,-1}, D\right)$ to the $\operatorname{kernel} \operatorname{ker}\left(d: \mathcal{E}^{p, 0} \rightarrow \mathcal{E}^{p, 1}\right)$, and as $D, d$ anticommute, the differential $D$ on the kernels is identified with the differential $-D$ on $\mathcal{E}^{p,-1}$. We note however that the change of sign does not affect the cohomology, so $H_{\mathcal{E}}^{*}(X, \mathcal{V})$ is isomorphic to the cohomology of $\left(\mathcal{E}^{*,-1}(X, \mathcal{V}), D\right)$ as claimed.

To capture the large scale geometry of a space $X$ we need to complete the $\mathcal{E}^{p, q}(X, \mathcal{V})$ bicomplex. Generalised Reiter sequences and asymptotically invariant means will appear in suitable completions which will be introduced in the following section.

\section{Generalised completions}

Let $\mathcal{E}$ be a vector space equipped with a countable family of seminorms $\|\cdot\|_{i}$ separating the points of $\mathcal{E}$. We will call such a space a pre-Fréchet space. We have in mind that $\mathcal{E}=\mathcal{E}^{p, q}(X, \mathcal{V})$, for some $p, q, X, G$ and $\mathcal{V}$, equipped with the $R$-norms as $R$ ranges over the natural numbers.

If $\mathcal{E}$ is not complete then one constructs the classical completion $\overline{\mathcal{E}}$ of $\mathcal{E}$ as follows. Let $E_{\mathrm{cs}}$ denote the space of Cauchy sequences in $\mathcal{E}$ (that is, sequences which are Cauchy with respect to each semi-norm), and let $E_{0}$ denote the space of sequences in $\mathcal{E}$ which converge to 0 . Then the completion of $\mathcal{E}$ is precisely the quotient space $E_{\mathrm{cs}} / E_{0}$. As the topology of $\mathcal{E}$ is given by a countable family of seminorms, this completion is a Fréchet space.

In this section we will define and study two generalised completions which are somewhat larger than the classical one. 
Definition 3.1 The quotient completion of $\mathcal{E}$, denoted $\mathcal{E}_{Q}$ is the quotient space $E / E_{0}$ where $E$ denotes the space of bounded sequences in $\mathcal{E}$ and $E_{0}$ denotes the space of sequences in $\mathcal{E}$ which converge to 0 . The family of seminorms on $\mathcal{E}$ yields a family of seminorms on $E$ and hence on the quotient $\mathcal{E}_{Q}$. The weak-* completion of $\mathcal{E}$, denoted $\mathcal{E}_{W}$ is the double dual of $\mathcal{E}$. The family of seminorms on $\mathcal{E}$ gives rise to a family of seminorms on $\mathcal{E}_{W}$.

We will adopt the convention that a class $\psi$ in the quotient completion $\mathcal{E}_{Q}$ may be represented by a sequence of functions $\psi_{n}$, where each $\psi_{n} \in \mathcal{E}$.

Let $I_{Q}$ denote the inclusion of $\mathcal{E}$ in $\mathcal{E}_{Q}$ as the space of constant sequences and let $I_{W}$ be the natural inclusion of $\mathcal{E}$ in its double dual $\mathcal{E}_{W}$. The space $\mathcal{E}$ is not assumed to be complete, but the maps $I_{Q}, I_{W}$ extend to an embedding of the classical completion $\overline{\mathcal{E}}$ in $\mathcal{E}_{Q}, \mathcal{E}_{W}$ respectively, and indeed $\mathcal{E}_{Q}, \mathcal{E}_{W}$ are isomorphic to the quotient and weak-* completions of $\overline{\mathcal{E}}$.

Since the space $\mathcal{E}$ need not be a normed space, we recall some basic theory of duals of Fréchet spaces. For simplicity we assume that the seminorms on $\mathcal{E}$ are monotonic, that is, $\|\cdot\|_{i} \leq\|\cdot\|_{j}$ for $i<j$, this being easy to arrange.

For $\alpha \in \mathcal{E}^{*}$, we can define $\|\alpha\|^{i}=\sup \left\{|\langle\alpha, \phi\rangle| \mid\|\phi\|_{i} \leq 1\right\}$. We note that $\|\alpha\|^{i}$ takes values in $[0, \infty]$, and $\|\cdot\|^{i} \geq\|\cdot\|^{j}$ for $i<j$. The condition that $\alpha$ is continuous is the condition that $\|\alpha\|^{i}$ is finite for some $i$. For any sequence $r_{1}, r_{2}, \ldots$ the set $\left\{\alpha \in \mathcal{E}^{*} \mid\|\alpha\|^{i}<r_{i}\right.$ for some $\left.i\right\}$ is a neighbourhood of 0 , and every neighbourhood of 0 contains such a set. Hence these sets determine the topology on $\mathcal{E}^{*}$.

Having equipped $\mathcal{E}^{*}$ with this topology, we can then form the space $\mathcal{E}^{* *}$ of continuous linear functionals on $\mathcal{E}^{*}$. A linear functional $\eta$ on $\mathcal{E}^{*}$ is continuous if for all $i$, setting $\|\eta\|_{i}=\sup \left\{|\langle\eta, \alpha\rangle| \mid\|\alpha\|^{i} \leq 1\right\}$ we have $\|\eta\|_{i}<\infty$.

The space $\mathcal{E}_{W}=\mathcal{E}^{* *}$ will be equipped with the weak-* topology. It follows by the Banach-Alaoglu theorem that all bounded subsets of $\mathcal{E}_{W}$ are relatively compact. In the language of Bourbaki, if $A \subseteq \mathcal{E}_{W}$ is bounded, that is, there exists a sequence $r_{i}$ such that $\|\eta\|_{i} \leq r_{i}$ for all $i$, then $A$ is contained in the polar of $\left\{\alpha \in \mathcal{E}^{*} \mid \exists i,\|\alpha\|^{i} \leq 1 / r_{i}\right\}$, which is compact.

Remark From an abstract perspective, the weak-* completion $\mathcal{E}_{W}$ is a natural way to enlarge $\mathcal{E}$. On the other hand, from the point of view of explicitly constructing elements, the quotient completion $\mathcal{E}_{Q}$ is more tractable.

Definition 3.2 We say that a short exact sequence $0 \rightarrow \mathcal{E} \stackrel{\iota}{\rightarrow} \mathcal{E}^{\prime} \stackrel{\pi}{\rightarrow} \mathcal{E}^{\prime \prime} \rightarrow 0$ of locally convex topological vector spaces is topologically exact if the map $\iota$ is topologically injective and $\pi$ is an open map. 
Note that if the spaces are complete then the requirements that $\iota$ is topologically injective and $\pi$ is open are automatic by the open mapping theorem.

Proposition 3.3 Let $\mathcal{E}, \mathcal{E}^{\prime}$ be pre-Fréchet spaces. Then a continuous map $T: \mathcal{E} \rightarrow \mathcal{E}^{\prime}$ induces maps $T^{Q}: \mathcal{E}_{Q} \rightarrow \mathcal{E}_{Q}^{\prime}$ and $T^{W}: \mathcal{E}_{W} \rightarrow \mathcal{E}_{W}^{\prime}$. Moreover this process is functorial and exact, that is, it takes short topologically exact sequences to short exact sequences.

Proof For the quotient completion, continuity of the map $T: \mathcal{E} \rightarrow \mathcal{E}^{\prime}$ guarantees that applying $T$ to each term of a bounded sequence $\phi_{n}$ in $\mathcal{E}$ we obtain a bounded sequence $T \phi_{n}$ in $\mathcal{E}^{\prime}$. If $\phi_{n} \rightarrow 0$ then $T \phi_{n} \rightarrow 0$ by continuity, hence we obtain a map $T^{Q}: \mathcal{E}_{Q} \rightarrow \mathcal{E}_{Q}^{\prime}$. It is clear that this respects compositions.

Now suppose $0 \rightarrow \mathcal{E} \stackrel{\iota}{\rightarrow} \mathcal{E}^{\prime} \stackrel{\pi}{\rightarrow} \mathcal{E}^{\prime \prime} \rightarrow 0$ is a short topologically exact sequence. If $\iota^{Q}$ vanishes on a class $\phi \in \mathcal{E}_{Q}$ then evaluating $\iota$ on a sequence $\phi_{n}$ representing $\phi$ we see that $\iota \phi_{n} \rightarrow 0$. Since $\iota$ is topologically injective, $\phi_{n} \rightarrow 0$. Hence $\phi=0$ and we have shown that $\iota^{Q}$ is injective.

Similarly if $\phi^{\prime} \in \mathcal{E}_{Q}^{\prime}$ with $\pi^{Q} \phi^{\prime}=0$ then $\pi \phi_{n}^{\prime} \rightarrow 0$. The map $\pi$ induces a map $\mathcal{E}^{\prime} / \mathfrak{L} \rightarrow \mathcal{E}^{\prime \prime}$ which is an isomorphism of pre-Fréchet spaces, hence the image of $\phi_{n}^{\prime}$ in the quotient $\mathcal{E}^{\prime} / \iota \mathcal{E}$ tends to 0 . That is, there exists a bounded sequence $\psi_{n}^{\prime}$ in $\iota \mathcal{E}$ such that $\phi_{n}^{\prime}-\psi_{n}^{\prime} \rightarrow 0$. We have $\psi_{n}^{\prime}=\iota \psi_{n}$ for some $\psi_{n} \in \mathcal{E}$, and since $\psi_{n}$ is a bounded sequence by topological injectivity of $\iota$, it represents a class $\psi$ in $\mathcal{E}_{Q}$. Then $\phi^{\prime}=\iota^{Q} \psi$ in $\mathcal{E}_{Q}^{\prime}$, hence we deduce that $\phi^{\prime}$ is in the image of $\iota^{Q}$.

Finally, for surjectivity of $\pi^{Q}$ we note that if $\phi^{\prime \prime} \in \mathcal{E}_{O}^{\prime \prime}$ then there exists a sequence $\phi_{n}^{\prime}$ such that $\phi_{n}^{\prime \prime}=\pi \phi_{n}^{\prime}$. By openness of $\pi$, the sequence $\phi_{n}^{\prime}$ can be chosen to be bounded as required.

In the case of the weak-* completion, the maps $T^{W}, \iota^{W}$ and $\pi^{W}$ are simply the double duals of $T, \iota$ and $\pi$. Passing to double duals respects composition, and the hypotheses that $\iota$ is topologically injective and $\pi$ is open ensures that the corresponding sequence of classical completions is exact, whence exactness of the double duals is standard.

We now give a connection between the two completions.

Proposition 3.4 Let $\omega$ be a non-principal ultrafilter on $\mathbb{N}$. Then for any pre-Fréchet space $\mathcal{E}$ there is a linear map $e_{\omega}: \mathcal{E}_{Q} \rightarrow \mathcal{E}_{W}$ satisfying $\left\langle e_{\omega}(\phi), \alpha\right\rangle=\lim _{\omega}\left\langle\alpha, \phi_{n}\right\rangle$ for all $\alpha$. Moreover $I_{W}=e_{\omega} \circ I_{Q}$ for all $\omega$ and for $T: \mathcal{E} \rightarrow \mathcal{E}^{\prime}$ we have $e_{\omega} \circ T^{Q}=T^{W} \circ e_{\omega}$. 
Proof Let $\phi$ denote an element of $\mathcal{E}_{Q}$ represented by a bounded sequence $\phi_{n}$ of elements of $\mathcal{E}$. We regard this sequence as a bounded map $\Phi: \mathbb{N} \rightarrow \mathcal{E}$. Regarding $\mathcal{E}$ as a subspace of its double dual $\mathcal{E}_{W}$, the closure of the range of this map is compact in the weak-* topology on $\mathcal{E}_{W}$ by the Banach-Alaoglu theorem. By the universal property of the Stone-Čech compactification it follows that $\Phi$ extends to a map $\bar{\Phi}: \beta \mathbb{N} \rightarrow \mathcal{E}_{W}$ which is continuous with respect to the weak-* topology on $\mathcal{E}_{W}$. Note that if $\phi_{n} \rightarrow 0$ then $\bar{\Phi}$ is identically 0 on $\partial \beta \mathbb{N}$, in particular it vanishes at $\omega$. We therefore define $e_{\omega}(\phi)=\bar{\Phi}(\omega)$ to obtain a well defined map $e_{\omega}: \mathcal{E}_{Q} \rightarrow \mathcal{E}_{W}$.

By continuity of $\bar{\Phi}$, for each $\alpha \in \mathcal{E}^{*}$, we obtain a continuous function on $\beta \mathbb{N}$ defined as $\langle\bar{\Phi}(\cdot), \alpha\rangle$. This is the extension to $\beta \mathbb{N}$ of the bounded function $n \mapsto\left\langle\alpha, \phi_{n}\right\rangle$, hence evaluating at $\omega$ we have

$$
\left\langle e_{\omega}(\phi), \alpha\right\rangle=\langle\bar{\Phi}(\omega), \alpha\rangle=\lim _{\omega}\left\langle\alpha, \phi_{n}\right\rangle .
$$

The fact that $e_{\omega} \circ T^{Q}=T^{W} \circ e_{\omega}$ is now easily verified as

$$
\left\langle e_{\omega}\left(T^{Q} \phi\right), \alpha\right\rangle=\lim _{\omega}\left\langle\alpha, T \phi_{n}\right\rangle=\lim _{\omega}\left\langle T^{*} \alpha, \phi_{n}\right\rangle=\left\langle e_{\omega}(\phi), T^{*} \alpha\right\rangle=\left\langle T^{W} e_{\omega}(\phi), \alpha\right\rangle
$$

for all $\alpha \in \mathcal{E}^{*}$. The compatibility of $e_{\omega}$ with the inclusion maps $I_{Q}, I_{W}$ is simply the observation that the extension to $\beta \mathbb{N}$ of a constant sequence is again constant.

We are now in a position to define our controlled cohomology theories.

\section{Controlled cohomology}

For $p \geq 0, q \geq-1$, let $\mathcal{E}_{Q}^{p, q}(X, \mathcal{V})$ denote the quotient completion of $\mathcal{E}^{p, q}(X, \mathcal{V})$, and let $\mathcal{E}_{W}^{p, q}(X, \mathcal{V})$ denote the weak-* completion of $\mathcal{E}^{p, q}(X, \mathcal{V})$. As $(D, d)$ are continuous anti-commuting differentials, the extensions of these to the completions (which we will also denote by $D, d$ ) are again anti-commuting differentials, hence taking $p, q \geq 0$ we have bicomplexes $\left(\mathcal{E}_{Q}^{p, q}(X, \mathcal{V}),(D, d)\right)$ and $\left(\mathcal{E}_{W}^{p, q}(X, \mathcal{V}),(D, d)\right)$.

Definition 4.1 For $\sim=Q$ or $W$, the $\sim-$ controlled cohomology of $X$ with coefficients in $\mathcal{V}$, denoted $H_{\sim}^{*}(X, \mathcal{V})$ is the cohomology of the totalisation of the bicomplex $\mathcal{E}_{\sim}^{p, q}(X, \mathcal{V}), p, q \geq 0$.

Since the splitting $s$ is continuous it extends to the completions and we deduce that the augmented rows of the completed bicomplexes are exact. This gives rise to the following. 
Corollary 4.2 The cohomologies $H_{Q}^{*}(X, \mathcal{V})$ and $H_{W}^{*}(X, \mathcal{V})$ are isomorphic, respectively, to the cohomologies of the cochain complexes $\left(\mathcal{E}_{Q}^{*,-1}(X, \mathcal{V}), D\right)$ and $\left(\mathcal{E}_{W}^{*,-1}(X, \mathcal{V}), D\right)$.

The argument is identical to Corollary 2.7.

We note that the extension of $s$ to the completions ensures that taking the kernel of $d: \mathcal{E}^{p, 0} \rightarrow \mathcal{E}^{p, 1}$ and then completing (in either way), yields the same result as first completing and then taking the kernel; one obtains the completion of $\mathcal{E}^{p,-1}$. The corresponding statement for $D$ would be false. The kernel of $D: \mathcal{E}_{Q}^{0, q} \rightarrow \mathcal{E}_{Q}^{1, q}$ will typically be much larger than the completion of the kernel of $D: \mathcal{E}^{0, q} \rightarrow \mathcal{E}^{1, q}$, and similarly for $\mathcal{E}_{W}$. We will study these kernels in Section 8, where we will use them to define the asymptotically invariant cohomology theories.

We now make a connection between the three cohomology theories $H_{\mathcal{E}}, H_{Q}, H_{W}$.

Theorem 4.3 For each non-principal ultrafilter $\omega$ on $\mathbb{N}$ the inclusions

$$
I_{Q}: \mathcal{E}^{p, q}(X, \mathcal{V}) \hookrightarrow \mathcal{E}_{Q}^{p, q}(X, \mathcal{V}) \quad \text { and } \quad I_{W}: \mathcal{E}^{p, q}(X, \mathcal{V}) \hookrightarrow \mathcal{E}_{W}^{p, q}(X, \mathcal{V})
$$

together with the map $e_{\omega}: \mathcal{E}_{Q}^{p, q}(X, \mathcal{V}) \rightarrow \mathcal{E}_{W}^{p, q}(X, \mathcal{V})$ defined in Proposition 3.4 induce a commutative diagram at the level of cohomology:

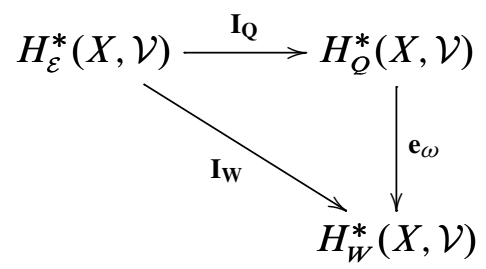

Moreover the kernels $\operatorname{ker} \mathbf{I}_{\mathbf{Q}}$ and $\operatorname{ker} \mathbf{I}_{\mathrm{w}}$ are equal, that is, a cocycle in $\mathcal{E}^{p, q}(X, \mathcal{V})$ is a coboundary in $\mathcal{E}_{Q}^{p, q}(X, \mathcal{V})$ if and only if it is a coboundary in $\mathcal{E}_{W}^{p, q}(X, \mathcal{V})$.

Proof The existence of the maps at the level of cohomology follows from the fact that $D, d$ commute with the inclusion maps and with each of the maps $e_{\omega}$. The diagram commutes at the level of cochains by Proposition 3.4. It is then immediate that $\operatorname{ker} \mathbf{I}_{\mathbf{Q}} \subseteq \operatorname{ker} \mathbf{I}_{\mathrm{w}}$. It remains to prove that if $\phi$ is a cocycle in $\mathcal{E}^{p, q}(X, \mathcal{V})$ with $I_{W} \phi$ a coboundary, then $I_{Q} \phi$ is also a coboundary.

By exactness of the rows, every cocycle in $\mathcal{E}^{p, q}(X, \mathcal{V})$ is cohomologous to an element of $\mathcal{E}^{p+q, 0}(X, \mathcal{V})$, hence without loss of generality we may assume that $q=0$. Moreover any cocycle in $\mathcal{E}^{p, 0}(X, \mathcal{V})$ is $d \phi$ for some $\phi$ in $\mathcal{E}^{p,-1}(X, \mathcal{V})$, and the images of $d \phi$ 
under $I_{Q}, I_{W}$ will be coboundaries if and only if $I_{Q} \phi, I_{W} \phi$ are coboundaries in the completions of the complex $\left(\mathcal{E}^{p,-1}(X, \mathcal{V}), D\right)$.

Suppose that $I_{W} \phi$ is a coboundary, that is viewing $\phi$ as an element of the double dual $\mathcal{E}_{W}^{p,-1}$, there exists $\psi$ in $\mathcal{E}_{W}^{p-1,-1}$ such that $D \psi=\phi$. We appeal to density of $\mathcal{E}^{p-1,-1}$ in $\mathcal{E}_{W}^{p-1,-1}$ to deduce that there is a bounded net $\theta_{\lambda}$ in $\mathcal{E}^{p-1,-1}$ converging to $\psi$ in the weak-* topology. By continuity of $D$ we have that $D \theta_{\lambda} \rightarrow D \psi=\phi$. As $D \theta_{\lambda}$ and $\phi$ lie in $\mathcal{E}^{p,-1}$, this converges in the weak topology on $\mathcal{E}^{p,-1}$. In any locally convex topological vector space, a convex set is closed in the locally convex topology if and only if it is closed in the associated weak topology. Hence (as the locally convex topology of $\mathcal{E}^{p,-1}$ is metrizable) there is a sequence $\theta_{n}$ of convex combinations of elements of the net $\theta_{\lambda}$ such that $D \theta_{n}$ converges to $\phi$ in the $R$-semi-norm topology on $\mathcal{E}^{p,-1}$. Thus, letting $\theta$ denote the element of $\mathcal{E}_{Q}$ represented by the sequence $\theta_{n}$, we have $D \theta=I_{Q} \phi$ in $\mathcal{E}_{Q}^{p,-1}$, so $I_{Q} \phi$ is a coboundary, as required.

\section{Group cohomology revisited}

In this section we consider the case where our space is a discrete group acting on itself by left multiplication and equipped with a proper left invariant metric. We show that in this case the controlled cohomology of $G$ can be identified with the standard group cohomology for suitably completed coefficients.

We say that an $X$-module $\mathcal{V}=(V,\|-\|$, Supp $)$ is non-degenerate if the subspace $V_{c}$ of compactly supported elements of $V$ is dense in $V$. We remark that for every $X$-module $\mathcal{V}$ the module $\mathcal{V}_{c}=\left(\bar{V}_{c},\|-\|\right.$, Supp) is a non-degenerate submodule, and moreover $H_{\sim}^{p}\left(X, \mathcal{V}_{c}\right)=H_{\sim}^{p}(X, \mathcal{V})$ for $\sim=\mathcal{E}, Q, W$.

Since $\mathcal{V}$ is an $X$-module $V$ is a Banach space and so too are its completions $V_{Q}$ and $V_{W}$. Furthermore a linear isometric action of $G$ on $V$ extends to a linear isometric action on each of these. We adopt our usual convention that $V \sim$ denotes either of these completions. Let $\left(C^{p}(G, V \sim), D\right)$, denote the homogeneous bar resolution computing the classical group cohomology with coefficients in $V \sim$.

Theorem 5.1 Let $G$ be a group acting on itself by left translation, and equipped with a proper left-invariant metric. Let $\mathcal{V}=(V,\|\cdot\|$, Supp) be a non-degenerate $G-$ module. Then there is an isomorphism of the cochain complexes $\left(C^{p}\left(G, V_{\sim}\right), D\right)$ and $\left(\mathcal{E}_{\sim}^{p,-1}(G, \mathcal{V}), D\right)$ inducing an isomorphism $H^{p}\left(G, V_{\sim}\right) \cong H_{\sim}^{p}(G, \mathcal{V})$, for $\sim=Q$ or $W$.

Proof For $\sim$ denoting $Q$ or $W$, set $\mathcal{V}_{\sim}^{\varnothing}=\left(V_{\sim},\|-\|\right.$, Supp $\left.^{\varnothing}\right)$, where Supp ${ }^{\varnothing}$ is the support function that assigns the empty set to every element of $V \sim$. 
We will show that $C^{p}\left(G, V_{\sim}\right)=\mathcal{E}^{p,-1}\left(G, \mathcal{V}_{\sim}^{\varnothing}\right) \cong \mathcal{E}_{\sim}^{p,-1}(G, \mathcal{V})$.

It is immediate that $\mathcal{E}^{p,-1}\left(G, \mathcal{V}_{\sim}^{\varnothing}\right) \subseteq C^{p}\left(G, V_{\sim}\right)$. Conversely, for every cochain $\phi \in C^{p}\left(G, V_{\sim}\right),\|\phi\|_{R}$ is finite for every $R$, since the $R$-neighbourhood of the diagonal contains only finitely many $G$-orbits, by properness of the metric. The condition of controlled supports is vacuous for $\mathcal{V}_{\sim}^{\varnothing}$ and this gives the reverse inclusion.

By definition, $\mathcal{E}^{p,-1}\left(G, \mathcal{V}_{\sim}^{\varnothing}\right) \cong \lim _{R} \ell^{\infty}\left(\Delta_{R}^{p}, V_{\sim}\right)^{G}$. Since $G$ has only finitely many orbits in $\Delta_{R}^{p}$, we have that

$$
\lim _{R} \ell^{\infty}\left(\Delta_{R}^{p}, V_{\sim}\right)^{G} \cong \lim _{R} \bigoplus_{G \backslash \Delta_{R}^{p}} V_{\sim} \cong \lim _{R} \bigoplus_{G \backslash \Delta_{R}^{p}}\left(V_{c}\right) \sim \lim _{R}\left(\bigoplus_{G \backslash \Delta_{R}^{p}} V_{c}\right)_{\sim}
$$

where we use that $V_{c}$ is dense in $V$, and that completions commute with direct sums. We now use the fact that the structure maps in the inverse system are surjective to conclude that

$$
\lim _{R}\left(\bigoplus_{G \backslash \Delta_{R}^{p}} V_{c}\right)_{\sim} \cong\left(\lim _{R} \bigoplus_{G \backslash \Delta_{R}^{p}} V_{c}\right)_{\sim} \cong\left(\lim _{R} \ell^{\infty}\left(\Delta_{R}^{p}, V_{c}\right)^{G}\right)_{\sim} .
$$

Since $G$ has only finitely many orbits on $\Delta_{R}^{p}$ the controlled support condition in $\mathcal{E}^{p,-1}(G, \mathcal{V})$ is precisely that cochains take values in $V_{c}$, hence

$$
\left(\lim _{\leftarrow} \ell^{\infty}\left(\Delta_{R}^{p}, V_{c}\right)^{G}\right)_{\sim} \cong \mathcal{E}_{\sim}^{p,-1}(G, \mathcal{V}),
$$

as required.

In the case of a group equipped with a proper metric the theorem tells us that the completion of the cochain complex can be obtained simply by completing the coefficients. This will not be true when the metric is not proper, nor will it hold in the general case of a metric space unless the associated group action is cocompact.

Now let $G$ be a countable discrete group equipped with a proper left invariant metric and let $\mathcal{J}$ be defined by $\mathcal{J}\left(g_{0}, g_{1}\right)=\delta_{g_{1}}-\delta_{g_{0}}$. In [3] we noted that amenability is characterised in classical (as opposed to bounded) cohomology by the vanishing of $[\mathcal{J}]$ as an element of $H^{1}\left(G,\left(\ell_{0}^{1}(G)\right)^{* *}\right)$, where $\ell_{0}^{1}(G)$ denotes the subspace of $\ell^{1}(G)$ consisting of functions which sum to 0 . Applying the above theorem we conclude that amenability is also characterised in terms of $H_{W}$.

Corollary 5.2 Let $G$ be a countable discrete group equipped with a proper left invariant metric and let $\mathcal{J} \in \mathcal{E}^{1,-1}\left(G, \ell_{0}^{1}(G)\right)$ be defined by $\mathcal{J}\left(g_{0}, g_{1}\right)=\delta_{g_{1}}-\delta_{g_{0}}$. Then $G$ is amenable if and only if $\left[I_{W} \mathcal{J}\right]=0$ in $H_{W}^{1}\left(G, \ell_{0}^{1}(G)\right)$. 
The analogue of this result in the context for the quotient completion is a special case of Theorem 7.3.

\section{Asymptotically invariant means}

An invariant mean on a group $G$ is a functional $\mu$ on $\ell^{\infty}(G)$ with total mass 1 which is invariant under the group action. Regarding $\mu$ as a 0 -cochain for the inhomogeneous bar resolution of $G$ over $\left(\ell^{\infty}(G)\right)^{*}$, the invariance condition is the assertion that $\mu$ is in fact a cocycle. Switching to the homogeneous picture and applying Theorem 5.1 we see that the invariance condition is equivalent to regarding $\mu$ as a cocycle in $\mathcal{E}_{W}^{0,-1}\left(G, \ell^{1}(G)\right)$. For our purposes it is then convenient to express the normalisation condition using a map on coefficients, in the spirit of the results in [3]. To this end we will consider the short exact sequence of coefficients:

$$
0 \rightarrow \ell_{0}^{1}(X) \stackrel{\iota}{\rightarrow} \ell^{1}(X) \stackrel{\pi}{\rightarrow} \mathbb{C} \rightarrow 0 .
$$

The first question we need to address is what it means for this to be a short exact sequence of $X$-modules. We begin with the concept of a morphism of coefficient modules. Let $X$ be a metric space, $G$ be a group acting by isometries on $X$ and let $\mathcal{U}=\left(U,|\cdot|_{U}, \operatorname{Supp}_{U}\right)$ and $\mathcal{V}=\left(V,|\cdot|_{V}, \operatorname{Supp}_{V}\right)$ be $G$-equivariant $X$-modules.

Definition 6.1 A $G$-equivariant $X$-morphism from $\mathcal{U}$ to $\mathcal{V}$ is an equivariant bounded linear map $\Psi: U \rightarrow V$ for which there exists $S \geq 0$ such that for all $u \in U$, $\operatorname{Supp}_{V}(\Psi(u)) \subseteq B_{S}\left(\operatorname{Supp}_{U}(u)\right)$. When the group action is clear from the context, in particular when $G$ is trivial, we will simply refer to this as an $X$-morphism.

An $X$-morphism $\Psi$ is said to be a monomorphism if it is injective and if there exists $T \geq 0$ such that for all $u \in U, \operatorname{Supp}_{U}(u) \subseteq B_{T}\left(\operatorname{Supp}_{V}(\Psi(u))\right)$.

An $X$-morphism $\Psi$ is said to be an epimorphism if it is surjective and there exists $M \geq$ 0 such that for all $R \geq 0$ there exists $S \geq 0$ such that for all $v \in V$ if $\operatorname{Supp}_{V}(v) \subseteq B_{R}(x)$ then there exists $u \in \Psi^{-1}(v)$ such that $\|u\|_{U} \leq M\|v\|_{V}$ and $\operatorname{Supp}_{U}(u) \subseteq B_{S}(x)$.

An $X$-morphism $\Psi$ is said to be an isomorphism if it is both an epimorphism and a monomorphism.

We adopt the convention that the term morphism refers to an $X$-morphism when both the space $X$ and the group $G$ are clear from the context.

It is straightforward to show that an $X$-morphism $\Psi: \mathcal{U} \rightarrow \mathcal{V}$ induces a continuous linear map $\Psi_{*}: \mathcal{E}^{p, q}(X, \mathcal{U}) \rightarrow \mathcal{E}^{p, q}(X, \mathcal{V})$ commuting with both differentials. This extends to give maps on both completed bicomplexes, $\mathcal{E}_{\sim}^{p, q}(X, \mathcal{U}) \rightarrow \mathcal{E}_{\sim}^{p, q}(X, \mathcal{V})$. 
Given a space $X$, and a group $G$ acting by isometries on $X$, a short exact sequence of $X$-modules is a short exact sequence of Banach spaces

$$
0 \rightarrow U \stackrel{\iota}{\rightarrow} V \stackrel{\pi}{\rightarrow} W \rightarrow 0
$$

each with the structure of a $G$-equivariant $X$-module, and where $\iota$ is a monomorphism of $X$-modules and $\pi$ is an epimorphism.

Example 6.2 Consider the following short exact sequence.

$$
0 \rightarrow \ell_{0}^{1}(X) \stackrel{\iota}{\rightarrow} \ell^{1}(X) \stackrel{\pi}{\rightarrow} \mathbb{C} \rightarrow 0
$$

For a $G$-space $X$ we regard these Banach spaces as $G$-modules in the natural way, where $G$ is regarded as acting trivially on $\mathbb{C}$. The function spaces are equipped with their usual support functions $\operatorname{Supp}(f)=\overline{\{x \in X \mid f(x) \neq 0\}}$ and $\mathbb{C}$ is equipped with the trivial support function $\operatorname{Supp}(\lambda)=\varnothing$ for all $\lambda \in \mathbb{C}$. We will show that this is a short exact sequence of $X$-modules.

The map $\iota$ is the standard "forgetful" inclusion of $\ell_{0}^{1}(X)$ into $\ell^{1}(X)$ and is easily seen to be a monomorphism. The map $\pi$ is the summation map and this is an epimorphism. To see this we argue as follows: since the support of any $\lambda \in \mathbb{C}$ is empty it lies within $R$ of any point $x \in X$. We choose the scaled Dirac delta function $\lambda \delta_{x} \in \ell^{1}(X)$ which clearly maps to $\lambda$, has norm $|\lambda|$ and $\operatorname{Supp}\left(\lambda \delta_{x}\right)=\{x\}$, so putting $M=1$ and $S=0$ satisfies the conditions.

Note that the constant function $\mathbf{1} \in \mathcal{E}^{0,-1}(X, \mathbb{C})$ taking the value 1 is a cocycle. Hence (applying $I_{Q}, I_{W}$ respectively) it represents a class $\left[\mathbf{1}_{Q}\right] \in H_{Q}^{0}(X, \mathbb{C})$, and another class $\left[\mathbf{1}_{W}\right] \in H_{Q}^{0}(X, \mathbb{C})$.

As in the previous section, consider the case of a group $G$ equipped with a proper left invariant metric. The map $\pi: \ell^{1}(G) \rightarrow \mathbb{C}$ induces a map $\pi_{*}: \mathcal{E}_{W}^{0,-1}\left(G, \ell^{1}(G)\right) \rightarrow$ $\mathcal{E}_{W}^{0,-1}(G, \mathbb{C})$ and, as discussed above, an invariant mean on the group is an element $\mu$ in $\mathcal{E}_{W}^{0,-1}\left(G, \ell^{1}(G)\right)$ such that $D \mu=0$ and $\pi_{*}(\mu)=\mathbf{1}_{W}$.

This motivates the following definition.

Definition 6.3 Let $X$ be a metric space equipped with an isometric action of a group $G$. An equivariant asymptotically invariant mean for $X$ is an element $\mu$ in $\mathcal{E}_{W}^{0,-1}\left(X, \ell^{1}(X)\right)$ such that $D \mu=0$ and $\pi_{*}(\mu)=\mathbf{1}_{W}$. In the special case when $G$ is the trivial group we simply call this an asymptotically invariant mean.

For a group $G$ equipped with a proper left invariant metric an equivariant asymptotically invariant mean is just an invariant mean, however this does not hold in general and we make the following definition. 
Definition 6.4 Let $G$ be a group equipped with a left invariant metric d (which we do not assume to be proper). We say that the pair $(G, d)$ is metrically amenable if it admits an equivariant asymptotically invariant mean.

We will consider this further in Section 10 in our discussion of Guentner's theorem concerning box spaces (see Roe [13]).

We conclude this section by establishing the existence of the long exact sequence in cohomology.

Proposition 6.5 A short exact sequence of $X$-modules induces a short exact sequence of bicomplexes for $\mathcal{E}, \mathcal{E}_{Q}$ and $\mathcal{E}_{W}$. Hence, by the snake lemma, we obtain long exact sequences in cohomology for $H_{\sim}^{*}(X,-)$, for each decoration $\sim=\mathcal{E}, Q$ or $W$.

Proof Suppose we are given a short exact sequence of $X$-modules

$$
0 \rightarrow \mathcal{U} \stackrel{\iota}{\rightarrow} \mathcal{V} \stackrel{\pi}{\rightarrow} \mathcal{W} \rightarrow 0 .
$$

We will show that the sequence

$$
0 \rightarrow \mathcal{E}^{p, q}(X, \mathcal{U}) \stackrel{\iota_{*}}{\rightarrow} \mathcal{E}^{p, q}(X, \mathcal{V}) \stackrel{\pi_{*}}{\longrightarrow} \mathcal{E}^{p, q}(X, \mathcal{W}) \rightarrow 0
$$

is topologically exact.

The map $\iota$ has closed range by exactness, so it is topologically injective by the open mapping theorem. Topological injectivity of $\iota_{*}$ then follows directly.

Exactness at the middle term follows from the observation that if $\pi_{*}(\phi)=0$ then $\phi=\iota \circ \phi^{\prime}$ for some function $\phi^{\prime}: X^{p+1} \times X^{q+1} \rightarrow U$, where $\phi^{\prime}$ is uniquely defined by injectivity of $\iota$. We need to verify that $\phi^{\prime}$ is an element of $\mathcal{E}^{p, q}(X, \mathcal{U})$. Topological injectivity of $\iota$ and boundedness of $\phi$ yields the required norm estimates, whereas the support condition is satisfied because $\iota$ is a monomorphism, hence $\operatorname{Supp}_{U}\left(\phi^{\prime}\right) \subseteq$ $B_{T}\left(\operatorname{Supp}_{V}\left(\iota \circ \phi^{\prime}\right)\right)=B_{T}\left(\operatorname{Supp}_{V}(\phi)\right)$ for some $T \geq 0$.

Surjectivity of $\pi_{*}$ follows from the definition of an epimorphism: Given $\phi \in \mathcal{E}^{p, q}(X, \mathcal{W})$, for each $R>0$ there exists $S>0$ such that $(\mathbf{x}, \mathbf{y}) \in \Delta_{R} \leq R$ implies that the support $\operatorname{Supp}_{W}(\phi(\mathbf{x}, \mathbf{y})) \subseteq B_{S}\left(x_{i}\right), B_{S}\left(y_{j}\right)$ for all $i, j$. Since $\pi$ is an epimorphism, there exists $M, T>0$ such that for each $(\mathbf{x}, \mathbf{y})$ there exists an element of $V$, which we denote $\phi^{\prime}(\mathbf{x}, \mathbf{y})$ such that $\left\|\phi^{\prime}(\mathbf{x}, \mathbf{y})\right\|_{V} \leq M\|\phi(\mathbf{x}, \mathbf{y})\|_{W}$ and $\operatorname{Supp}_{V}\left(\phi^{\prime}(\mathbf{x}, \mathbf{y})\right) \subseteq$ $B_{T}\left(x_{i}\right), B_{T}\left(y_{j}\right)$ for each $i, j$, so $\phi^{\prime}$ is of controlled supports and has finite $R$-norms as required. These estimates for the $R$-norms also ensure that $\pi_{*}$ is open.

Proposition 3.3 allows us to extend these maps to the completions $\mathcal{E}_{Q}$ and $\mathcal{E}_{W}$ to obtain short exact sequences for both the $\mathcal{E}_{Q}$ and $\mathcal{E}_{W}$ bicomplexes. It is now immediate 
from the snake lemma that for each decoration $\sim=\mathcal{E}, Q, W$ there is a connecting homomorphism $D$ inducing a long exact sequence in cohomology:

$0 \longrightarrow H_{\sim}^{0}(X, \mathcal{U}) \longrightarrow H_{\sim}^{0}(X, \mathcal{V}) \longrightarrow H_{\sim}^{0}(X, \mathcal{W})$

$$
\stackrel{D}{\longrightarrow} H_{\sim}^{1}(X, \mathcal{U}) \longrightarrow H_{\sim}^{1}(X, \mathcal{V}) \longrightarrow H_{\sim}^{1}(X, \mathcal{W}) \longrightarrow \cdots
$$

This completes the proof.

\section{A cohomological characterisation of property A}

We will use the following definition of property A, which is equivalent to Yu's original definition for spaces of bounded geometry (see Higson and Roe [6]).

Definition 7.1 A metric space $X$ is said to have property A if for each $x \in X$ and each $n \in \mathbb{N}$ there is an element $f_{n}(x) \in \operatorname{Prob}(X)$ with

(1) a sequence $S_{n}$ such that $\operatorname{Supp}\left(f_{n}(x)\right) \subseteq B_{S_{n}}(x)$ and

(2) for any $R \geq 0,\left\|f_{n}\left(x_{1}\right)-f_{n}\left(x_{0}\right)\right\|_{\ell^{1}} \rightarrow 0$ as $n \rightarrow \infty$, uniformly on the set $\left\{\left(x_{0}, x_{1}\right) \mid d\left(x_{0}, x_{1}\right) \leq R\right\}$.

We refer to the sequence $f_{n}$ as a generalised Reiter sequence for $X$. When $X$ is a countable discrete group equipped with a proper left invariant metric, and each $f_{n}$ is equivariant, then this gives us a classical Reiter sequence [11]. By equivariance, the first condition in the definition then reduces to the usual finite support condition and is thus independent of the metric. Similarly, the second condition reduces to pointwise convergence.

As an application of the long exact sequence we give our first cohomological characterisation of Yu's property A in terms of the vanishing of the Johnson class in the controlled cohomology $H_{\sim}^{1}\left(X, \ell_{0}^{1}(X)\right)$.

Let $X$ be a metric space (as usual we have in the background a group acting by isometries on $X$, but our applications in this section will assume that the action is trivial). Recall the short exact sequence of $X$-modules introduced in Example 6.2

$$
0 \rightarrow \ell_{0}^{1}(X) \stackrel{\iota}{\rightarrow} \ell^{1}(X) \stackrel{\pi}{\rightarrow} \mathbb{C} \rightarrow 0 .
$$

As usual let $\mathbf{1} \in \mathcal{E}^{0,-1}(X, \mathbb{C})$ denote the constant function 1 on $X$.

Lemma 7.2 Suppose the action of $G$ on $X$ is trivial. Then $X$ has property $A$ if and only if $\mathcal{E}_{Q}^{0,-1}\left(X, \ell^{1}(X)\right)$ contains an element $\phi$ such that $D \phi=0$ and $\pi_{*} \phi=\mathbf{1}_{Q}$. 
Proof Any generalised Reiter sequence $f_{n}$ for $X$ provides (as in Definition 7.1) an element of $\mathcal{E}_{Q}$ with the required properties: the fact that $f_{n}(x)$ is a probability measure ensures that $\pi f_{n}(x)=1$ for all $x, n$, that is $\pi_{*} f=I_{Q} 1$. The other hypotheses of Definition 7.1 are precisely the assertions that $f$ is of controlled supports and that $D f=0$ in $\mathcal{E}_{Q}^{1,-1}\left(X, \ell^{1}(X)\right)$.

Conversely, given an element $\phi \in \mathcal{E}_{Q}^{-1}\left(X, \ell^{1}(X)\right)$ such that $D \phi=0$ and $\pi_{*} \phi=I_{Q} \mathbf{1}$, represented by a sequence $\phi_{n}$, we set

$$
f_{n}(x)(z)=\frac{\left|\phi_{n}(x)(z)\right|}{\left\|\phi_{n}(x)\right\|_{\ell^{1}}} .
$$

Since $\pi \phi_{n}(x)=1$ for all $x, n$ we have

$$
\frac{1}{\left\|\phi_{n}(x)\right\|_{\ell^{1}}} \leq 1
$$

As an element of $\ell^{1}(X), f_{n}(x)$ has the same supports as $\phi_{n}(x)$, in particular $f_{n}$ is of controlled supports for all $n$. The verification that $\left\|f_{n}\left(x_{1}\right)-f_{n}\left(x_{0}\right)\right\|_{\ell^{1}}$ tends to 0 uniformly on $\left\{\left(x_{0}, x_{1}\right) \mid d\left(x_{0}, x_{1}\right) \leq R\right\}$ follows from the fact that $D \phi=0$ and the estimate $\frac{1}{\left\|\phi_{n}(x)\right\|_{\ell}} \leq 1$.

Now recall the long exact sequences for $H_{\sim}$, where $\sim=Q$ or $W$ :

$$
\begin{aligned}
0 \longrightarrow H_{\sim}^{0}\left(X, \ell_{0}^{1}(X)\right) \stackrel{\iota_{*}}{\longrightarrow} H_{\sim}^{0}\left(X, \ell^{1}(X)\right) \stackrel{\pi_{*}}{\longrightarrow} H_{\sim}^{0}(X, \mathbb{C}) \\
\stackrel{D}{\longrightarrow} H_{\sim}^{1}\left(X, \ell_{0}^{1}(X)\right) \stackrel{\iota_{*}}{\longrightarrow} H_{\sim}^{1}\left(X, \ell^{1}(X)\right) \stackrel{\pi_{*}}{\longrightarrow}
\end{aligned}
$$

The connecting map $D$ yields a class $D\left[\mathbf{1}_{Q}\right]$ in $H_{Q}^{1}\left(X, \ell_{0}^{1}(X)\right)$, and a class $D\left[\mathbf{1}_{W}\right]$ in $H_{W}^{1}\left(X, \ell_{0}^{1}(X)\right)$.

The classical Johnson class on a group $G$ is defined by the 1-cocycle $\mathcal{J}\left(g_{0}, g_{1}\right)=$ $\delta_{g_{1}}-\delta_{g_{0}}$, where $\delta_{g}$ denotes the Dirac delta function which takes the value 1 at $g$ and 0 elsewhere. By analogy we define the element $\mathcal{J}^{1,0} \in \mathcal{E}^{1,0}\left(X, \ell_{0}^{1}(X)\right)$ by $\mathcal{J}^{1,0}\left(\left(x_{0}, x_{1}\right), y\right)=\delta_{x_{1}}-\delta_{x_{0}}$. This element is a cocycle so by applying $I_{Q}, I_{W}$ we obtain elements $\left[\mathcal{J}_{Q}^{1,0}\right] \in H_{Q}^{1}\left(X, \ell_{0}^{1}(X)\right)$ and $\left[\mathcal{J}_{W}^{1,0}\right] \in H_{W}^{1}\left(X, \ell_{0}^{1}(X)\right)$ which we refer to as the Johnson classes for $X$. We note that the class of $\mathcal{J}^{1,0}$ in $H_{\mathcal{E}}\left(X, \ell_{0}^{1}(X)\right)$ is the image of [1] under the connecting homomorphism, since we may pull back the function 1 to the Dirac element $x \mapsto \delta_{x}$ and applying the coboundary map to this we obtain $\mathcal{J}^{1,0}$. By applying $I_{Q}$ and $I_{W}$ we observe that $\left[\mathcal{J}_{Q}^{1,0}\right]=D\left[\mathbf{1}_{Q}\right]$ and $\left[\mathcal{J}_{W}^{1,0}\right]=D\left[\mathbf{1}_{W}\right]$.

We are now ready to give a cohomological characterisation of the existence of an equivariant asymptotically invariant mean. In the case where the group action is trivial, 
we characterise property A both in terms of the existence of an asymptotically invariant mean and in cohomological terms.

Theorem 7.3 Let $X$ be a discrete metric space equipped with an isometric action of a group $G$. Then the following are equivalent:

(1) $\left[\mathbf{1}_{Q}\right] \in \operatorname{Im} \pi_{*}$ in $H_{Q}^{0}(X, \mathbb{C})$.

(2) $\left[\mathcal{J}_{Q}^{1,0}\right]=D\left[\mathbf{1}_{Q}\right]=0$ in $H_{Q}^{1}\left(X, \ell_{0}^{1}(X)\right)$.

(3) $\left[\mathcal{J}_{W}^{1,0}\right]=D\left[\mathbf{1}_{W}\right]=0$ in $H_{W}^{1}\left(X, \ell_{0}^{1}(X)\right)$.

(4) $\left[\mathbf{1}_{W}\right] \in \operatorname{Im} \pi_{*}$ in $H_{W}^{0}(X, \mathbb{C})$.

(5) $X$ admits an equivariant asymptotically invariant mean.

If the group $G$ acts trivially on $X$ then these conditions are all equivalent to property $A$ for the metric space $X$.

Proof Conditions (1) and (2) are equivalent by exactness of the long exact sequence in cohomology, while (2) is equivalent to (3) by Theorem 4.3. Conditions (3) and (4) are equivalent by a further application of the long exact sequence (this time for the weak-* completion). The equivalence of (4) and (5) is immediate from the definition of asymptotically invariant mean. To prove the final statement we note that (1) is equivalent to property A by Lemma 7.2.

\section{Asymptotically invariant cohomology}

We pause for a moment to recall the classical definition of bounded cohomology for a group. One first takes the homogeneous bar resolution wherein the $k$-dimensional cochains consist of all bounded functions from $G^{k+1}$ to $\mathbb{C}$. This cochain complex is exact so has trivial cohomology. This is exhibited by taking a basepoint splitting which is induced by the map $G^{k} \rightarrow G^{k+1}$ given by inserting the basepoint as an additional (first) co-ordinate. Now one takes the $G$-invariant part of this complex, where $G$ acts diagonally and $\mathbb{C}$ is equipped with the trivial action of $G$. Since the splitting is not equivariant the corresponding cochain complex is not necessarily exact. When the group $G$ is amenable one can average the splitting over orbits using the invariant mean, and this produces an equivariant splitting which therefore kills the cohomology in dimensions greater than or equal to 1 .

As discussed in Section 1 the bounded cochain complex may be regarded as the bottom row of an augmented complex obtained by taking the kernels of the vertical differentials 
in degree 0 . In this section and the following we will carry out an analogous process for property $\mathrm{A}$. In this section we will construct the asymptotically invariant cohomology of a space as the analogue of bounded cohomology. Replacing the classical (split) cochain complex by the first row of the $\mathcal{E}_{\sim}$ bicomplex, $\left(\mathcal{E}_{\sim}^{0, q}, d\right)$, (which is acyclic since $\left(\mathcal{E}^{0, q}, d\right)$ is acyclic by Proposition 2.6) we take the kernels under the vertical differential $D$ to produce a new cochain complex, which is the analogue of taking the $G$-invariant parts in group cohomology.

The splitting $s$ of the horizontal differential $d$ does not restrict to this cochain complex leaving room for interesting cohomology. In the following section we will show that if the space $X$ has property A one can asymptotically average the splitting $s$ to obtain a splitting of the asymptotically invariant complex. Hence we will deduce that if $X$ has property A then the asymptotically invariant cohomology vanishes in all dimensions greater than or equal to 1 .

Definition 8.1 We say that an element $\phi$ of $\mathcal{E}_{O}^{0, q}$ (respectively $\mathcal{E}_{W}^{0, q}$ ) is asymptotically invariant if $D \phi=0$ in $\mathcal{E}_{Q}^{1, q}$ (respectively $\mathcal{E}_{W}^{1, q}$ ). Let $\mathcal{E}_{Q A}^{q}$ and $\mathcal{E}_{W A}^{q}$, denote the spaces of asymptotically invariant elements in $\mathcal{E}_{Q}^{0, q}$ and $\mathcal{E}_{W}^{0, q}$ respectively. We note as usual that this is defined for $q \geq-1$.

For notational convenience when considering elements of $\mathcal{E}^{0, q}$ we will suppress the parentheses around the single $x$ variable, writing $\phi\left(x,\left(y_{0}, \ldots, y_{q}\right)\right)$.

The term asymptotically invariant is motivated by the case of $\mathcal{E}_{Q}^{0, q}$. An element of $\mathcal{E}_{Q}^{0, q}$ is asymptotically invariant if it is represented by a sequence $\phi_{n}: X \times X^{q+1} \rightarrow V$ which is asymptotically invariant in the $x$ variable the following sense: for all $R>0$ the difference $\phi_{n}\left(x_{1}, \mathbf{y}\right)-\phi_{n}\left(x_{0}, \mathbf{y}\right)$ tends to zero uniformly on $\Delta_{R}^{2} \times X^{q+1}$.

We remark that it is essential that we first complete the complex $\mathcal{E}$ and then take the kernels of $D$, not the other way around. If we were to take the kernel of $D: \mathcal{E}^{0, q} \rightarrow \mathcal{E}^{1, q}$ we would get functions $\phi\left(x,\left(y_{0}, \ldots, y_{q}\right)\right)$ which are constant in the $x$ variable, that is, we would have invariant rather than asymptotically invariant elements. The kernel of $D: \mathcal{E}_{\sim}^{0, q} \rightarrow \mathcal{E}_{\sim}^{1, q}$ will typically be much larger than the completion of these $x$-invariant functions.

We now make the following elementary observation.

Proposition 8.2 The differential $d$ maps $\mathcal{E}_{Q A}^{q}(X, \mathcal{V})$ to $\mathcal{E}_{Q A}^{q+1}(X, \mathcal{V})$, and $\mathcal{E}_{W A}^{q}(X, \mathcal{V})$ to $\mathcal{E}_{W A}^{q+1}(X, \mathcal{V})$. Hence $\left(\mathcal{E}_{Q A}^{q}(X, \mathcal{V}), d\right)$ and $\left(\mathcal{E}_{W A}^{q}(X, \mathcal{V}), d\right)$ are complexes.

Proof This is immediate from anti-commutativity of the differentials $D, d$. 
Recall that there is a splitting $s: \mathcal{E}^{0, q} \rightarrow \mathcal{E}^{0, q-1}$ extending to both generalised completions. To see that $s$ does not necessarily map the asymptotically invariant subcomplex into itself consider the following example.

Example 8.3 For a metric space $X$ we define a Johnson element $\mathcal{J}^{0,1} \in \mathcal{E}^{0,1}\left(X, \ell^{1}(X)\right)$ by $\mathcal{J}^{0,1}\left(x,\left(y_{0}, y_{1}\right)\right)=\delta_{y_{1}}-\delta_{y_{0}}$. Since $\mathcal{J}^{0,1}$ is independent of $x, D \mathcal{J}^{0,1}=0$, so $\mathcal{J}_{Q}^{0,1}=I_{Q} \mathcal{J}^{0,1}$ lies in $\mathcal{E}_{Q A}^{1}$, and $\mathcal{J}_{W}^{0,1}=I_{W} \mathcal{J}^{0,1}$ lies in $\mathcal{E}_{W A}^{1}$. However

$$
\begin{aligned}
D s \mathcal{J}^{0,1}\left(\left(x_{0}, x_{1}\right),\left(y_{0}\right)\right) & =s \mathcal{J}^{0,1}\left(x_{1},\left(y_{0}\right)\right)-s \mathcal{J}^{0,1}\left(x_{0},\left(y_{0}\right)\right) \\
& =\left(\delta_{y_{0}}-\delta_{x_{1}}\right)-\left(\delta_{y_{0}}-\delta_{x_{0}}\right) \\
& =\delta_{x_{0}}-\delta_{x_{1}}
\end{aligned}
$$

which has $\ell^{1}$-norm equal to 2 for all $x_{0} \neq x_{1}$. Hence $D s \mathcal{J}_{Q}^{0,1}=I_{Q} D s \mathcal{J}^{0,1} \neq 0$ and $D s \mathcal{J}_{W}^{0,1}=I_{W} D s \mathcal{J}^{0,1} \neq 0$, so neither $s \mathcal{J}_{Q}^{0,1}$ nor $s \mathcal{J}_{W}^{0,1}$ is asymptotically invariant.

We can now define the asymptotically invariant cohomology.

Definition 8.4 For $\sim$ equal to either of the decorations $Q$ or $W$, the $\sim$-asymptotically invariant cohomology of $X$ with coefficients in the module $\mathcal{V}$ is the cohomology of the complex $\left(\mathcal{E}_{\sim A}^{*}(X, \mathcal{V}), d\right)$. It is denoted $H_{\sim A}^{*}(X, \mathcal{V})$. Where the completion used is clear from the context we will refer to this as simply the asymptotically invariant cohomology of $X$.

\section{Lemma 8.5 The augmentation maps}

$$
\mathcal{E}_{Q A}^{q}(X, \mathcal{V}) \hookrightarrow \mathcal{E}_{Q}^{0, q}(X, \mathcal{V}) \quad \text { and } \quad \mathcal{E}_{W A}^{q}(X, \mathcal{V}) \hookrightarrow \mathcal{E}_{W}^{0, q}(X, \mathcal{V})
$$

induce maps on cohomology

$$
H_{Q A}^{q}(X, \mathcal{V}) \rightarrow H_{Q}^{q}(X, \mathcal{V}) \quad \text { and } \quad H_{W A}^{q}(X, \mathcal{V}) \rightarrow H_{W}^{q}(X, \mathcal{V}),
$$

which are isomorphisms for $q=0$, and are injective for $q=1$.

Proof Since $D$ vanishes on $\mathcal{E}_{Q A}^{q}(X, \mathcal{V})$, the differential on the asymptotically invariant complex is the restriction of the differential $D+d$ on the totalisation of the bicomplex, so the augmentation map induces a map $H_{Q A}^{q}(X, \mathcal{V}) \rightarrow H_{Q}^{q}(X, \mathcal{V})$. In degree 0 every cocycle is non-trivial, and if $\phi \in \mathcal{E}_{Q}^{0,0}$ is a cocycle then $D \phi=0$ so $\phi$ is asymptotically invariant whence the map is an isomorphism. In degree 1 , if $\phi \in \mathcal{E}_{Q}^{0,1}(X, \mathcal{V})$ is a coboundary in the totalisation of the bicomplex then there is an element $\psi$ of $\mathcal{E}_{Q}^{0,0}(X, \mathcal{V})$ such that $(D+d) \psi$ is $(0 \oplus \phi)$ in $\mathcal{E}_{Q}^{1,0}(X, \mathcal{V}) \oplus \mathcal{E}_{Q}^{0,1}(X, \mathcal{V})$. That is $D \psi=0$, so $\psi$ is an element of $\mathcal{E}_{Q A}^{0}(X, \mathcal{V})$, and $d \psi=\phi$. Hence $\phi$ is also a coboundary in $\mathcal{E}_{Q A}^{1}(X, \mathcal{V})$. Hence the inclusion of $\mathcal{E}_{Q A}^{1}(X, \mathcal{V})$ into $\mathcal{E}_{Q}^{0,1}(X, \mathcal{V})$ gives an injection of cohomology.

The proof for $\mathcal{E}_{W}$ is identical. 
Now we restrict to the case where $G$ is trivial, and $\mathcal{V}$ is $\ell_{0}^{1}(X)$. The Johnson element $\mathcal{J}^{0,1}\left(x,\left(y_{0}, y_{1}\right)\right)=\delta_{y_{1}}-\delta_{y_{0}}$ in $\mathcal{E}^{0,1}(X, \mathcal{V})$ gives classes $\left[\mathcal{J}_{Q}^{0,1}\right] \in H_{Q A}^{1}(X, \mathcal{V})$ and $\left[\mathcal{J}_{W}^{0,1}\right] \in H_{Q A}^{1}(X, \mathcal{V})$. Applying the augmentation map we obtain elements of $H_{Q}^{1}(X, \mathcal{V})$ and $H_{W}^{1}(X, \mathcal{V})$. As noted above

$$
\operatorname{Ds}\left(\mathcal{J}^{0,1}\right)\left(\left(x_{0}, x_{1}\right), y\right)=\delta_{x_{0}}-\delta_{x_{1}}=-\mathcal{J}^{1,0}\left(\left(x_{0}, x_{1}\right), y\right),
$$

so $\mathcal{J}^{1,0}$ is cohomologous to $\mathcal{J}^{0,1}$ in the totalisation of $\mathcal{E}^{*, *}\left(X, \ell_{0}^{1}(X)\right)$. From this it is immediate that we have $\left[\mathcal{J}_{Q}^{0,1}\right]=\left[\mathcal{J}_{Q}^{1,0}\right]=D\left[\mathbf{1}_{Q}\right]$ in $H_{Q}^{1}\left(X, \ell_{0}^{1}(X)\right)$ and $\left[\mathcal{J}_{W}^{0,1}\right]=$ $\left[\mathcal{J}_{W}^{1,0}\right]=D\left[\mathbf{1}_{W}\right]$ in $H_{W}^{1}\left(X, \ell_{0}^{1}(X)\right)$.

We thus obtain the following theorem.

Theorem 8.6 Let $X$ be a metric space with trivial $G$ action. Then the following are equivalent:

(1) $X$ has property $A$.

(2) $\left[\mathcal{J}_{Q}^{0,1}\right]=0$ in $H_{Q A}^{1}\left(X, \ell_{0}^{1}(X)\right)$.

(3) $\left[\mathcal{J}_{W}^{0,1}\right]=0$ in $H_{W A}^{1}\left(X, \ell_{0}^{1}(X)\right)$.

Proof By Lemma 8.5, for $\sim$ denoting $Q$ or $W,\left[\mathcal{J}_{\sim}^{0,1}\right]$ is zero in $H_{\sim A}^{1}\left(X, \ell_{0}^{1}(X)\right)$ if and only if it is zero in $H_{\sim}^{1}\left(X, \ell_{0}^{1}(X)\right)$, and we have seen that its image is equal to $\left[\mathcal{J}_{\sim}^{1,0}\right]$. By Theorem 7.3, this vanishes if and only if $X$ has property A.

\section{Vanishing theorems}

Throughout this section we will consider a metric space $X$ with trivial group action.

We have seen that the map $s$ does not in general split the coboundary map $d$ in the complexes $\mathcal{E}_{Q A}^{*}$ and $\mathcal{E}_{W A}^{*}$, however if $X$ has property A then we can use the generalised Reiter sequence in the case of the quotient completion, and the asymptotically invariant mean in the case of the weak-* completion, to asymptotically average $s \phi$. Having done so we will obtain a splitting for the asymptotically invariant complexes, demonstrating the vanishing of the cohomology.

We will make use of the following convolution operator.

Definition 9.1 For $f \in \mathcal{E}^{p,-1}\left(X, \ell^{1}(X)\right)$ and $\theta \in \mathcal{E}^{0, q}(X, V)$, define $f * \theta$ by

$$
(f * \theta)(\mathbf{x}, \mathbf{y})=\sum_{z} f(\mathbf{x})(z) \theta(z, \mathbf{y}) .
$$


We remark that as $\theta$ lies in the bottom row of the bicomplex, $\|\theta\|_{R}$ is constant in $R$, so we suppress the $R$ in the notation for brevity.

We make the following estimate:

$$
\begin{aligned}
\|f * \theta\|_{R} & \leq \sup _{\mathbf{x} \in \Delta_{R}^{p+1}, \mathbf{y} \in X^{q+1}} \sum_{z \in X}|f(\mathbf{x})(z)|\|\theta(z, \mathbf{y})\|_{V} \\
& \leq \sup _{\mathbf{x} \in \Delta_{R}^{p+1}} \sum_{z}|f(\mathbf{x},(z))|\|\theta\| \\
& =\|f\|_{R}\|\theta\| .
\end{aligned}
$$

This estimate shows that for each $f$ the map $\theta \mapsto f * \theta$ is continuous, and for each $\theta$ the map $f \mapsto f * \theta$ is continuous.

We note that $D(f * \phi)(\mathbf{x}, \mathbf{y})=\sum_{z} \sum_{i}(-1)^{i} f\left(\widehat{\mathbf{x}}_{i}\right)(z) \phi(z, \mathbf{y})=((D f) * \phi)(\mathbf{x}, \mathbf{y})$, by exchanging the order of summation.

Similarly $d(f * \phi)(\mathbf{x}, \mathbf{y})=(f * d \phi)(\mathbf{x}, \mathbf{y})$.

The convolution extends in an obvious way to the quotient completion. For $f \in$ $\mathcal{E}_{Q}^{q,-1}\left(X, \ell^{1}(X)\right), \phi \in \mathcal{E}_{Q}^{0, q}(X, V)$ we define $f * \phi \in \mathcal{E}_{Q}^{p, q}(X, V)$ by $(f * \phi)_{n}=f_{n} * \phi_{n}$. We note that if either of the sequences $f_{n}, \phi_{n}$ tends to 0 as $n \rightarrow \infty$, then $(f * \phi)_{n}$ tends to 0 by the above norm estimate. Hence the convolution is a well defined map

$$
\mathcal{E}_{Q}^{p,-1}\left(X, \ell^{1}(X)\right) \times \mathcal{E}_{Q}^{0, q}(X, V) \rightarrow \mathcal{E}_{Q}^{p, q}(X, V),
$$

that is, as an element of $\mathcal{E}_{Q}^{p, q}(X, V)$, the convolution $f * \phi$ does not depend on the choice of sequences representing $f, \phi$.

Since the convolution is defined term-by-term in $n$, the identities $D(f * \phi)=(D f) * \phi$ and $d(f * \phi)=f * d \phi$ carry over to the quotient completion.

We recall that by Lemma 7.2 property $\mathrm{A}$ is equivalent to the existence of an element $f$ of $\mathcal{E}_{Q}^{0,-1}\left(X, \ell^{1}(X)\right)$ with $D f=0$ and $\pi_{*}(f)=\mathbf{1}_{Q}$. Convolving with such an $f$ allows us to average the splitting $s \phi$ to get an asymptotically invariant element. We use this idea to prove the following theorem.

Theorem 9.2 If $X$ is a metric space satisfying Yu's property $A$, then the asymptotically invariant cohomology $H_{Q A}^{q}(X, \mathcal{V})$ is zero for every $q \geq 1$ and every $X$-module $\mathcal{V}$.

Proof Let $\phi \in \mathcal{E}_{Q A}^{q}(X, \mathcal{V})$ with $q \geq 1$. The element $\phi$ is represented by a sequence $\phi_{n}$ in $\mathcal{E}^{q}(X, \mathcal{V})$ and $s \phi$ is represented by the sequence

$$
s \phi_{n}\left(x,\left(y_{0}, \ldots, y_{q-1}\right)\right)=\phi_{n}\left(x,\left(x, y_{0}, \ldots, y_{q-1}\right)\right) .
$$


Since $D \phi=0$, the sequence $D \phi_{n}$ tends to zero, that is for all $R>0,\left\|D \phi_{n}\right\|_{R} \rightarrow 0$ as $n \rightarrow \infty$. By a diagonal argument, if $S_{n}$ is a sequence tending to infinity sufficiently slowly, then $\left\|D \phi_{n}\right\|_{S_{n}} \rightarrow 0$ as $n \rightarrow \infty$. We choose a sequence $S_{n}$ with this property.

Take a generalised Reiter sequence $f$ in $\mathcal{E}_{Q}^{0,-1}\left(X, \ell^{1}(X)\right)$ so that $D f=0$ and $\pi_{*}(f)=$ $\mathbf{1}_{Q}$, and let $f_{n}$ be a sequence representing $f$. If $f_{n}$ is a sequence representing $f$, then $f_{n}(x)+\left(1-\pi\left(f_{n}(x)\right)\right) \delta_{x}$ also represents $f$ and has sum 1, so without loss of generality we may assume that $\pi\left(f_{n}(x)\right)=1$ for all $x, n$.

By repeating the terms of the sequence $f_{n}$ we can arrange that $\operatorname{Supp}\left(f_{n}(x)\right) \subseteq B_{S_{n}}(x)$ for all $x, n$. Note that our choice of $f$ therefore depends on $S_{n}$ and hence on $\phi$.

As a remark in passing, we note that taking such a 'supersequence' of $f_{n}$ corresponds in some sense to taking a subsequence of $\phi_{n}$. If we were working in the classical completion $E_{\mathrm{cs}} / E_{0}$, then the subsequence would represent the same element of $E_{\mathrm{cs}} / E_{0}$, however for $\mathcal{E}_{Q}$ this need not be true.

For each $q^{\prime}$ we now define $s_{f}: \mathcal{E}_{Q}^{0, q^{\prime}}(X, V) \rightarrow \mathcal{E}_{Q}^{0, q^{\prime}-1}(X, V)$ by $s_{f} \psi=f * s \psi$. We first note that for any $\psi$ the element $s_{f} \psi$ is asymptotically invariant. This follows from asymptotic invariance of $f$, since $D s_{f} \phi=D(f * s \phi)=(D f) * s \phi=0$. Hence in fact we have a map $s_{f}: \mathcal{E}_{Q}^{0, q^{\prime}}(X, V) \rightarrow \mathcal{E}_{Q A}^{q^{\prime}-1}(X, V)$ which restricts to the asymptotically invariant complex.

We claim that for our given $\phi$ we have $\left(d s_{f}+s_{f} d\right) \phi=\phi$. We have $d s_{f} \phi=d(f * s \phi)=$ $f * d s \phi$, while $s_{f} d \phi=f * s d \phi$ by definition. Hence $\left(d s_{f}+s_{f} d\right) \phi=f *(d s+s d) \phi=$ $f * \phi$ since $d s+s d=1$. It thus remains to show that $f * \phi=\phi$. Notice that since $\sum_{z \in X} f_{n}(x)(z)=1$ we have $\phi_{n}(x, \mathbf{y})=\sum_{z \in X} f_{n}(x)(z) \phi_{n}(x, \mathbf{y})$, so we have

$$
\begin{aligned}
\left(f_{n} * \phi_{n}-\phi_{n}\right)(x, \mathbf{y}) & =\sum_{z \in X} f_{n}(x)(z)\left(\phi_{n}(z, \mathbf{y})-\phi_{n}(x, \mathbf{y})\right) \\
& =\sum_{z \in X} f_{n}(x)(z) D \phi_{n}((x, z), \mathbf{y}) .
\end{aligned}
$$

Taking norms we have $\left\|f_{n} * \phi_{n}-\phi_{n}\right\| \leq\left\|f_{n}\right\|\left\|D \phi_{n}\right\|_{S_{n}}$, since if $d(x, z)>S_{n}$ then $f_{n}(x)(z)$ vanishes. We know that $\left\|D \phi_{n}\right\|_{S_{n}} \rightarrow 0$ as $n \rightarrow \infty$, hence we conclude that $f * \phi-\phi=0$ in $\mathcal{E}_{Q A}^{q}(X, \mathcal{V})$.

We have shown that for every element $\phi \in \mathcal{E}_{Q A}^{q}(X, \mathcal{V})$ with $q \geq 1$, we can construct maps $s_{f}: \mathcal{E}_{Q A}^{q^{\prime}}(X, \mathcal{V}) \rightarrow \mathcal{E}_{Q A}^{q^{\prime}-1}(X, \mathcal{V})$ such that $\left(d s_{f}+s_{f} d\right) \phi=\phi$. (As noted above, $f$, and hence $s_{f}$, depend on the element $\phi$.) It follows that if $\phi$ is a cocycle then $\phi=\left(d s_{f}+s_{f} d\right) \phi=d s_{f} \phi$, so every cocycle is a coboundary. Thus we deduce that $H_{Q A}^{q}(X, \mathcal{V})=0$ for $q \geq 1$. 
We will now prove a corresponding result for the weak-* completion. The role of the generalised Reiter sequence $f_{n}$ in the previous argument will be replaced by an asymptotically invariant mean $\mu$ in $\mathcal{E}_{W}^{0,-1}\left(X, \ell^{1}(X)\right)$.

We begin by extending the convolutions to the weak-* completions. First we define $f * \phi$ for $f \in \mathcal{E}^{p,-1}\left(X, \ell^{1}(X)\right)$ and $\phi \in \mathcal{E}_{W}^{0, q}(X, V)$. This is defined via its pairing with an element $\alpha$ of $\mathcal{E}^{p, q}(X, V)^{*}$ :

$$
\langle f * \phi, \alpha\rangle=\left\langle\phi, \alpha_{f}\right\rangle \text {, where }\left\langle\alpha_{f}, \theta\right\rangle=\langle\alpha, f * \theta\rangle \text {, for all } \theta \in \mathcal{E}^{0, q}(X, V) .
$$

In other words the operator $\phi \mapsto f * \phi$ on $\mathcal{E}_{W}^{0, q}(X, V)$ is the double dual of the operator $\theta \mapsto f * \theta$ on $\mathcal{E}^{0, q}(X, V)$.

We have $\left|\left\langle\alpha_{f}, \theta\right\rangle\right| \leq\|\alpha\|_{R}\|f * \theta\|_{R} \leq\|\alpha\|_{R}\|f\|_{R}\|\theta\|$ for some $R$ (depending on $\alpha$ ). Hence for each $\alpha$ there exists $R$ such that

$$
|\langle f * \phi, \alpha\rangle| \leq\|\phi\|\|\alpha\|_{R}\|f\|_{R}
$$

so $f * \phi$ is a continuous linear functional.

We now want to further extend the convolution to define $\eta * \phi$ in $\mathcal{E}_{W}^{p, q}(X, V)$, for $\eta \in$ $\mathcal{E}_{W}^{p,-1}\left(X, \ell^{1}(X)\right)$ and $\phi \in \mathcal{E}_{W}^{0, q}(X, V)$. The definition is motivated by the requirement that $\left(I_{W} f\right) * \phi=f * \phi$. Hence for $\alpha$ in $\mathcal{E}^{p, q}(X, V)^{*}$ we will require

$$
\left\langle\left(I_{W} f\right) * \phi, \alpha\right\rangle=\langle f * \phi, \alpha\rangle .
$$

For $\phi \in \mathcal{E}_{W}^{0, q}(X, V), \alpha \in \mathcal{E}^{p, q}(X, V)^{*}$, define $\sigma_{\phi, \alpha} \in \mathcal{E}^{p,-1}\left(X, \ell^{1}(X)\right)^{*}$ by

$$
\left\langle\sigma_{\phi, \alpha}, f\right\rangle=\langle f * \phi, \alpha\rangle=\left\langle\phi, \alpha_{f}\right\rangle .
$$

The above inequalities ensure that $\sigma_{\phi, \alpha}$ is a continuous linear functional.

We observe that $f * \phi$ is determined by the property that $\langle f * \phi, \alpha\rangle=\left\langle\sigma_{\phi, \alpha}, f\right\rangle=$ $\left\langle I_{W} f, \sigma_{\phi, \alpha}\right\rangle$. We use this to give the general definition: For $\eta \in \mathcal{E}_{W}^{p,-1}\left(X, \ell^{1}(X)\right)$ and $\phi \in \mathcal{E}_{W}^{0, q}(X, V)$, we define $\eta * \phi$ in $\mathcal{E}_{W}^{p, q}(X, V)$ by

$$
\langle\eta * \phi, \alpha\rangle=\left\langle\eta, \sigma_{\phi, \alpha}\right\rangle
$$

for all $\alpha$ in $\mathcal{E}^{p, q}(X, V)^{*}$.

Lemma 9.3 For $\eta \in \mathcal{E}_{W}^{p,-1}\left(X, \ell^{1}(X)\right)$ and $\phi \in \mathcal{E}_{W}^{0, q}(X, \mathcal{V})$ we have $D(\eta * \phi)=$ $(D \eta) * \phi$ and $d(\eta * \phi)=\eta * d \phi$.

Proof The elements $D(\eta * \phi), d(\eta * \phi)$ are defined by their pairings with respectively $\alpha$ in $\mathcal{E}^{p+1, q}(X, \mathcal{V})^{*}$ and $\beta$ in $\mathcal{E}^{p, q+1}(X, \mathcal{V})^{*}$. These are given by pairing $\eta$ with respectively $\sigma_{\phi, D^{*} \alpha}$ and $\sigma_{\phi, d^{*} \beta}$. 
Since for $f \in \mathcal{E}^{p,-1}\left(X, \ell^{1}(X)\right)$ we have

$$
\text { and } \begin{aligned}
\left\langle\sigma_{\phi, D^{*} \alpha}, f\right\rangle & =\left\langle\phi,\left(D^{*} \alpha\right)_{f}\right\rangle \\
\left\langle\sigma_{\phi, d^{*} \beta}, f\right\rangle & =\left\langle\phi,\left(d^{*} \beta\right)_{f}\right\rangle,
\end{aligned}
$$

we must determine $\left(D^{*} \alpha\right)_{f}$ and $\left(d^{*} \beta\right)_{f}$. Pairing these with an element $\theta$ in $\mathcal{E}^{0, q}(X, \mathcal{V})$ we have

$$
\begin{aligned}
\left\langle\left(D^{*} \alpha\right)_{f}, \theta\right\rangle & =\langle\alpha, D(f * \theta)\rangle=\langle\alpha,(D f) * \theta\rangle, \\
\text { and } \quad\left\langle\left(d^{*} \beta\right)_{f}, \theta\right\rangle & =\langle\beta, d(f * \theta)\rangle=\langle\beta, f * d \theta\rangle .
\end{aligned}
$$

Hence $\left(D^{*} \alpha\right)_{f}=\alpha_{D f}$ and $\left(d^{*} \beta\right)_{f}=d^{*}\left(\beta_{f}\right)$, so we have $\sigma_{\phi, D^{*} \alpha}=D^{*} \sigma_{\phi, \alpha}$ and $\sigma_{\phi, d^{*} \beta}=\sigma_{d \phi, \beta}$. It follows that $D(\eta * \phi)=(D \eta) * \phi$ and $d(\eta * \phi)=\eta * d \phi$ as required.

Before proceeding with the proof of the vanishing theorem we first establish the following result.

Lemma 9.4 If $\eta \in \mathcal{E}_{W}^{0,-1}\left(X, \ell^{1}(X)\right)$ is in the image of $\mathcal{E}_{W}^{0,-1}\left(X, \ell_{0}^{1}(X)\right)$, and $\phi \in$ $\mathcal{E}_{W}^{0, q}(X, V)$ with $D \phi=0$ then $\eta * \phi=0$.

Proof The statement that $\eta * \phi=0$, amounts to the assertion that $\left\langle\eta, \sigma_{\phi, \alpha}\right\rangle=0$ for all $\alpha$ in $\mathcal{E}^{0, q}(X, V)^{*}$. Since the image of $I_{W}$ is dense in $\mathcal{E}_{W}^{0,-1}\left(X, \ell_{0}^{1}(X)\right)$ in the weak-* topology, it suffices to show that $\left\langle\sigma_{\phi, \alpha}, f\right\rangle=0$ for all $f \in \mathcal{E}^{0,-1}\left(X, \ell_{0}^{1}(X)\right)$. We note that

$$
\left\langle\sigma_{\phi, \alpha}, f\right\rangle=\langle f * \phi, \alpha\rangle=\left\langle\phi, \alpha_{f}\right\rangle .
$$

We will show that $\alpha_{f}$ is a 'boundary,' that is $\alpha_{f}$ is in the range of the map $D^{*}$. As $D \phi=0$ it will follow that the pairing is trivial.

We define a boundary map $\partial: \ell^{1}(X \times X) \rightarrow \ell_{0}^{1}(X)$ by

$$
(\partial H)\left(z_{0}\right)=\sum_{z_{1} \in X} H\left(z, z_{0}\right)-H\left(z_{0}, z\right) .
$$

Equivalently, we can write

$$
\partial H=\sum_{z_{0}, z_{1} \in X} H\left(z_{0}, z_{1}\right)\left(\delta_{z_{1}}-\delta_{z_{0}}\right) .
$$

We note that $\partial$ is surjective: For $h \in \ell_{0}^{1}(X)$ and $x$ in $X$, let $H\left(z_{0}, z_{1}\right)=h\left(z_{1}\right)$ if $z_{0}=x, z_{1} \neq x$ and let $H\left(z_{0}, z_{1}\right)=0$ otherwise. Then $\partial H=h$. We note that $\|H\|_{\ell^{1}} \leq\|h\|_{\ell^{1}}$, and $\operatorname{Supp}(H) \subseteq\{x\} \times \operatorname{Supp}(h)$. For each $x$, let $F(x)$ be the lift of 
$f(x)$ constructed in this way, so that $\|F(x)\|_{\ell^{1}} \leq\|f(x)\|_{\ell^{1}}$ for all $x$, and as $f$ is of controlled supports there exists $R$ such that if $F(x)\left(z_{0}, z_{1}\right) \neq 0$ then $z_{0}, z_{1} \in B_{R}(x)$. Writing $(\partial F)(x)=\partial(F(x))$, for $\theta \in \mathcal{E}^{0, q}(X, V)$, we have

$$
\left\langle\alpha_{f}, \theta\right\rangle=\langle\alpha, f * \theta\rangle=\langle\alpha,(\partial F) * \theta\rangle .
$$

Now compute $(\partial F) * \theta$. We have

$$
\begin{aligned}
((\partial F) * \theta)(x, \mathbf{y}) & =\sum_{z} \partial F(x)(z) \theta(z, \mathbf{y}) \\
& =\sum_{z, z_{0}, z_{1}} F(x)\left(z_{0}, z_{1}\right)\left(\delta_{z_{1}}(z)-\delta_{z_{0}}(z)\right) \theta(z, \mathbf{y}) \\
& =\sum_{z_{0}, z_{1}} F(x)\left(z_{0}, z_{1}\right) D \theta\left(\left(z_{0}, z_{1}\right), \mathbf{y}\right)
\end{aligned}
$$

We define $T_{F}: \mathcal{E}^{1, q}(X, V) \rightarrow \mathcal{E}^{0, q}(X, V)$ by

$$
\left(T_{F} \zeta\right)(x, \mathbf{y})=\sum_{z_{0}, z_{1}} F(x)\left(z_{0}, z_{1}\right) \zeta\left(\left(z_{0}, z_{1}\right), \mathbf{y}\right) .
$$

As $F(x)\left(z_{0}, z_{1}\right) \neq 0$ implies $z_{0}, z_{1}$ lie in the ball $B_{R}(x)$, we have the estimate

$$
\begin{aligned}
\left\|T_{F} \zeta\right\| & \leq \sup _{x \in X, \mathbf{y} \in X^{q+1}} \sum_{z_{0}, z_{1} \in X} \mid F(x)\left(z_{0}, z_{1}\right)\|\| \zeta\left(\left(z_{0}, z_{1}\right), \mathbf{y}\right) \|_{V} \\
& \leq \sup _{x \in X}\|F(x)\|_{\ell^{1}}\|\zeta\|_{R} \\
& \leq\|f\|\|\zeta\|_{R^{\prime}} .
\end{aligned}
$$

hence $T_{F}$ is continuous.

We conclude that

$$
\left\langle\alpha_{f}, \theta\right\rangle=\langle\alpha,(\partial F) * \theta\rangle=\left\langle\alpha, T_{F} D \theta\right\rangle=\left\langle D^{*} T_{F}^{*} \alpha, \theta\right\rangle
$$

for all $\theta$, hence $\alpha_{f}=D^{*} T_{F}^{*} \alpha$, so that

$$
\left\langle\phi, \alpha_{f}\right\rangle=\left\langle\phi, D^{*} T_{F}^{*} \alpha\right\rangle=\left\langle D \phi, T_{F}^{*} \alpha\right\rangle=0 .
$$

This completes the proof.

We now prove the vanishing theorem.

Theorem 9.5 If $X$ is a metric space satisfying Yu's property $A$, then the asymptotically invariant cohomology $H_{W A}^{q}(X, \mathcal{V})$ is zero for every $q \geq 1$ and every $X$-module $\mathcal{V}$.

Specifically, if $\mu$ is an asymptotically invariant mean then $s_{\mu} \phi=\mu * s \phi$ defines a splitting of the asymptotically invariant complex. 
Proof By Theorem 7.3, property A guarantees the existence of an asymptotically invariant mean $\mu$, that is an element $\mu$ in $\mathcal{E}_{W A}^{0,-1}$ such that $\pi_{*}(\mu)=0$.

We define $s_{\mu}: \mathcal{E}_{W}^{0, q}(X, \mathcal{V}) \rightarrow \mathcal{E}_{W}^{0, q-1}(X, \mathcal{V})$ by $s_{\mu} \phi=\mu * s \phi$. By Lemma 9.3 we have $D s_{\mu} \phi=D(\mu * s \phi)=(D \mu) * s \phi$. Since $\mu$ is asymptotically invariant $D \mu=0$, so $s_{\mu} \phi$ is also asymptotically invariant. Hence $s_{\mu}$ restricts to a map $s_{\mu}: \mathcal{E}_{W A}{ }^{0, q}(X, \mathcal{V}) \rightarrow$ $\mathcal{E}_{W A}{ }^{0, q-1}(X, \mathcal{V})$. We must now verify that $s_{\mu}$ is a splitting. By Lemma 9.3, and using the fact that $d s+s d=1$ we have

$$
\left(d s_{\mu}+s_{\mu} d\right) \phi=d(\mu * s \phi)+\mu * s d \phi=\mu * d s \phi+\mu * s d \phi=\mu * \phi .
$$

It thus remains to show that $\mu * \phi=\phi$.

Let $\delta$ denote the map $X \rightarrow \ell^{1}(X), x \mapsto \delta_{x}$. We have $\pi_{*}\left(I_{W} \delta\right)=1=\pi_{*}(\mu)$, so for $\eta=\delta-\mu$ we have $\pi_{*}(\eta)=0$. Hence $\eta$ is in the image of $\mathcal{E}_{W}^{0,-1}\left(X, \ell_{0}^{1}(X)\right)$. As $D \phi=0$, it follows from Lemma 9.4 that $\eta * \phi=0$. Thus $\mu * \phi=\left(I_{W} \delta\right) * \phi=\delta * \phi$. It is easy to see that convolution with $\delta$ yields the identity map on $\mathcal{E}^{0, q}(X, \mathcal{V})$, hence its double dual is again the identity map. Thus $\mu * \phi=\delta * \phi=\phi$ as required.

This completes the proof.

Combining Theorems 8.6, 9.2 and 9.5 we obtain the following.

Theorem 9.6 Let $X$ be a discrete metric space with trivial group action. Then the following are equivalent:

(1) $X$ has property $A$.

(2) $H_{Q A}^{q}(X, \mathcal{V})=0$ for all $q \geq 1$ and all $X$-modules $\mathcal{V}$.

(3) $\left[\mathcal{J}_{Q}^{0,1}\right]=0$ in $H_{Q A}^{1}\left(X, \ell_{0}^{1}(X)\right)$.

(4) $H_{W A}^{q}(X, \mathcal{V})=0$ for all $q \geq 1$ and all $X$-modules $\mathcal{V}$.

(5) $\left[\mathcal{J}_{W}^{0,1}\right]=0$ in $H_{W A}^{1}\left(X, \ell_{0}^{1}(X)\right)$.

\section{Non-property A spaces}

There are essentially three examples of spaces known not to have property A: expander sequences, box spaces of non-amenable groups and the union of finite cubes of all dimensions. The existing proofs are distinct in character; in this section we unify the latter two examples, while in a companion note [8], Ana Khukhro and the third author apply cohomological methods in the case of expanders. We first consider box spaces of non-amenable groups. This example, which is due to Guentner, was explored by Roe in [13]. 
Definition 10.1 Let $G$ be a countable residually finite group equipped with a proper left invariant metric $d$ valued in $\mathbb{Z}$. Let $X_{\lambda}=G / N_{\lambda}, \lambda \in \Lambda$ be a family of finite quotients of $G$, such that for any $\lambda, \mu \in \Lambda$, there exists $v \in \Lambda$ such that $N_{\nu} \leq N_{\mu} \cap N_{\lambda}$. We regard $\Lambda$ as a directed system by defining $\lambda<\mu$ when $N_{\lambda} \geq N_{\mu}$. We equip each of the quotients $X_{\lambda}$ with the quotient metric $d_{\lambda}$ and extend this to a metric on $\bigsqcup_{\lambda \in \Lambda} X_{\lambda}$ in the usual way, requiring that for each positive integer $K$, the set of all pairs $\left(\lambda, \lambda^{\prime}\right)$ for which $\lambda \neq \lambda^{\prime}$ and the distance from $X_{\lambda}$ to $X_{\lambda^{\prime}}$ is less than $K$, is finite. The disjoint union $X=\bigsqcup_{\lambda \in \Lambda} X_{\lambda}$ is said to be a box space for $G$ if the intersection of the kernels $N_{\lambda}$ is trivial.

For any $X_{\lambda}$ in the system we denote the natural map $G \rightarrow X_{\lambda}$ by $q_{\lambda}$ and the image of an element $g$ under $q_{\lambda}$ by $g_{\lambda}$. Similarly if $\lambda<\mu$ there is a natural map $q_{\lambda \mu}: X_{\mu} \rightarrow X_{\lambda}$ and for any $x \in X_{\mu}$ we denote $q_{\lambda \mu}(x)=x_{\lambda}$. Note that if $\lambda<\mu<v$ and $x \in X_{\nu}$ then $\left(x_{\mu}\right)_{\lambda}=x_{\lambda}$, and similarly, for $g \in G,\left(g_{\mu}\right)_{\lambda}=g_{\lambda}$.

Theorem 10.2 (Guentner and Roe [13, Proposition 11.39]) Let $G$ be a residually finite, finitely generated group and let $X$ be a box space for $G$. Then $G$ is amenable if and only if $X$ has property $A$.

Here we give a cohomological proof of this result which applies more generally to the situation where $G$ is a countable discrete group equipped with a proper left invariant metric. In Theorem 10.4 we will generalise this to the case of an arbitrary left invariant discrete metric by replacing the classical notion of amenability with the notion of metric amenability introduced in Definition 6.4 above.

Proof If $G$ is amenable then there exists $\phi=\left(\phi_{n}\right) \in \mathcal{E}_{Q}^{0,-1}\left(G, \ell^{1}(G)\right)$, with $D \phi=0$ and $\pi_{*}(\phi)=\mathbf{1}_{Q}$. We define a push forward map

$$
q_{*}: \mathcal{E}^{0,-1}\left(G, \ell^{1}(G)\right) \rightarrow \mathcal{E}^{0,-1}\left(X, \ell^{1}(X)\right)
$$

by

$$
q_{*} \psi(x)=x\left(q_{\lambda}\right)_{*} \psi(e)
$$

where $x \in X_{\lambda}$ and $\left(q_{\lambda}\right)_{*}$ is the push-forward map from $\ell^{1}(G)$ to $\ell^{1}\left(X_{\lambda}\right)$. Since $q_{*}$ is bounded we can extend it to the quotient completion. As $\pi$ is the map induced by the quotient to a point, $\pi_{*}\left(q_{*}(\phi)\right)=\mathbf{1}_{Q}$ by functoriality of the push forward.

For $x_{0}, x_{1} \in X_{\lambda}$, let $g_{0}, g_{1} \in G$ be pre-images with $d\left(g_{0}, g_{1}\right)=d\left(x_{0}, x_{1}\right)$. Then $D q_{*} \phi_{n}\left(x_{0}, x_{1}\right)=\left(q_{\lambda}\right)_{*} D \phi\left(g_{0}, g_{1}\right)$, so if $d\left(x_{0}, x_{1}\right) \leq R$ then $\left\|D q_{*} \phi_{n}\left(x_{0}, x_{1}\right)\right\|_{\ell^{1}} \leq$ $\left\|D \phi_{n}\right\|_{R}$. 
Enumerate the indexing set $\Lambda$ as $\lambda_{1}, \lambda_{2}, \ldots$ and let $F_{n}=\bigcup_{i \leq n} X_{\lambda_{i}}$. Now define $\xi_{n} \in \mathcal{E}^{0,-1}\left(X, \ell^{1}(X)\right)$ by averaging $q_{*} \phi$ over $F_{n}$, that is,

$$
\xi_{n}(x)=\frac{1}{\left|F_{n}\right|} \sum_{x^{\prime} \in F_{n}} q_{*} \phi_{n}\left(x^{\prime}\right)
$$

if $x \in F_{n}$ and $\xi_{n}(x)=q_{*} \phi_{n}(x)$ otherwise.

The distance between components $X_{\lambda_{n}}$ tends to infinity with $n$, hence for each $R$ and a sufficiently large $n$, if $d\left(x_{0}, x_{1}\right) \leq R$, then $x_{0}, x_{1}$ both lie in a common component or both lie in $F_{n}$. Hence $\left\|D \xi_{n}\right\|_{R} \leq\left\|D \phi_{n}\right\|_{R}$, so $\xi_{n}$ defines a cocycle $\xi \in \mathcal{E}_{Q}\left(X, \ell^{1}(X)\right)$. The averaging process does not affect $\pi_{*}$ so $\pi_{*}(\xi)=\mathbf{1}_{Q}$. We conclude that $X$ has property A.

For the reverse implication assume that $X$ has property A, so by Theorem 7.3 it admits an asymptotically invariant mean.

We will define a "pullback" map $\mathcal{E}^{p,-1}\left(X, \ell^{1}(X)\right) \rightarrow \mathcal{E}^{p,-1}\left(G, \ell^{1}(G)\right)$ by regarding $\psi \in \mathcal{E}^{p,-1}\left(X, \ell^{1}(X)\right)$ as a bounded function of $p+2$ variables (the additional variable is the module variable) and using an ultrafilter to extract a pointwise limit. First we set

$$
\phi_{\lambda}(\mathbf{g})(h)=\frac{1}{\left|X_{\lambda}\right|} \sum_{x \in X_{\lambda}} \psi\left(x \mathbf{g}_{\lambda}\right)\left(x h_{\lambda}\right)
$$

where for $\mathbf{g}=\left(g_{0}, \ldots, g_{p}\right), x \mathbf{g}_{\lambda}$ denotes $\left(x q_{\lambda}\left(g_{0}\right), \ldots, x q_{\lambda}\left(g_{p}\right)\right)$.

For each $\mathbf{g}, h$ we note that $\phi_{\lambda}(\mathbf{g})(h)$ is bounded in $\lambda$ and so we can define $\phi(\mathbf{g})(h)$ to be the ultrafilter limit. Moreover, $\|\phi\|_{R} \leq\|\psi\|_{R}$ for all $R$ since for $\mathbf{g} \in \Delta_{R}^{p+1}(G)$, $\mathbf{g}_{\lambda} \in \Delta_{R}^{p+1}\left(X_{\lambda}\right)$, and for any finite subset $F \subset G$, if $\lambda$ is sufficiently large then $q_{\lambda}$ is injective on $F$ by residual finiteness, so

$$
\sum_{h \in F}\left|\phi_{\lambda}(\mathbf{g})(h)\right| \leq \sum_{z \in X_{\lambda}} \frac{1}{\left|X_{\lambda}\right|} \sum_{x \in X_{\lambda}}\left|\psi\left(x \mathbf{g}_{\lambda}\right)(z)\right| \leq \frac{1}{\left|X_{\lambda}\right|} \sum_{x \in X_{\lambda}}\|\psi\|_{R}=\|\psi\|_{R} .
$$

Since $\psi$ is of controlled supports for any $R>0$ there exists $S>0$ such that if $\mathbf{x} \in \Delta_{R}^{p+1}(X)$ then for each $i, \operatorname{Supp}(\psi(x)) \subseteq B_{S}\left(x_{i}\right)$. Let $\mathbf{g} \in \Delta_{R}^{p+1}(G)$ and suppose that $h \notin B_{S}\left(g_{i}\right)$ for some $i$. For sufficiently large $\lambda$ the distance $d\left(h_{\lambda},\left(g_{i}\right)_{\lambda}\right)=$ $d\left(h, g_{i}\right)>S$, and so $d\left(x h_{\lambda}, x\left(g_{i}\right)_{\lambda}\right)>S$ for all $x$. Hence $\phi_{\lambda}(\mathbf{g}, h)=0$. It follows that the support of $\phi(g) \subseteq B_{S}\left(g_{i}\right)$ for each $i$, so $\phi$ is of controlled supports. It is easy to see that $\phi$ is equivariant.

Note that norm estimate above allows us to extend the pullback to the completed complexes and it is clear that the pullback commutes with the differential $D$ before, and hence after, completion. It follows that if $\psi$ is an asymptotically invariant mean for 
$X$ then its pullback $\phi$ is invariant. We now compute $\pi_{*}(\phi)$. By the controlled support condition for $S$ sufficiently large $\operatorname{Supp}(\phi(g)) \subseteq B_{S}(g)$ and $\operatorname{Supp}\left(\psi\left(g_{\lambda}\right)\right) \subseteq B_{S}\left(g_{\lambda}\right)$. If $\lambda$ is sufficiently large then $B_{S}(g)$ is isometric to $B_{S}\left(g_{\lambda}\right)$ so we obtain

$$
\sum_{h \in B_{S}(g)} \phi_{\lambda}(g)(h)=\frac{1}{\left|X_{\lambda}\right|} \sum_{x \in X_{\lambda}} \sum_{z \in B_{S}\left(g_{\lambda}\right)} \psi\left(x g_{\lambda}\right)(x z)=\frac{1}{\left|X_{\lambda}\right|} \sum_{x \in X_{\lambda}} \pi\left(\psi\left(x g_{\lambda}\right)\right)=1 .
$$

Taking the limit over $\lambda$ we see that $\pi(\phi(g))=1$ so the pullback of an asymptotically invariant mean on $X$ is an invariant mean on $G$.

We note that in the above proof, the key point at which properness of the metric was invoked was when we established that the pullback is compatible with $\pi$. We also use it implicitly when we assert that for all $g, g^{\prime} \in G$ there exists a $\lambda$ such that $d\left(g, g^{\prime}\right)=d\left(g_{\lambda}, g_{\lambda}^{\prime}\right)$.

Many examples of box spaces fail to embed uniformly in Hilbert space. In [9] Nowak gave an example of a space which does admit a uniform embedding in Hilbert space, but does not possess property A: let $X$ denote the disjoint union of the finite cubes $X_{n}=\{0,1\}^{n}$ each equipped with the $\ell^{1}$ metric, and extend this to a proper metric on $X$ insisting that the distance from $X_{n}$ to its complement tends to $\infty$ with $n$. Then $X$ does not have property A.

In Theorem 10.4 we will give a generalisation of Guentner's theorem and we will show that Nowak's example arises as an application of this result. To do so we identify the finite cubes as quotients of the group $G=\bigoplus_{i \in \mathbb{N}} \mathbb{Z}_{2}$, equipped with the word metric on the natural generators. We note that $G$ is amenable since it is an ascending union of finite groups, so we might expect the box space to have property A. As we will see the fact that it does not have property A follows from the observation that $G$ is not metrically amenable, as stated in Definition 6.4.

Lemma 10.3 Let $G$ be the group $\bigoplus_{i \in \mathbb{N}} \mathbb{Z}_{2}$ equipped with the word metric induced by its natural generating set. Then $G$ is not metrically amenable.

Proof Suppose that $\phi \in \mathcal{E}_{Q}^{0,-1}\left(G, \ell^{1}(G)\right)$ such that $\pi_{*}(\phi)=\mathbf{1}_{Q}$. We will show that $D \phi \neq 0$. For each $n$ and for each $\epsilon>0$ there is a finite subset $F$ such that $\left\|\left.\phi_{n}(e)\right|_{F^{c}}\right\|<\epsilon$ so that $\left\|\left.\phi_{n}(e)\right|_{F}\right\| \geq 1-\epsilon$. Since $F$ is finite, we may now choose a generator $s$ of $G$ such that $s F \cap F=\varnothing$. Then by equivariance $\phi_{n}(s)=s \phi_{n}(e)$ so 
$\left\|\left.\phi_{n}(s)\right|_{S F^{c}}\right\|<\epsilon$ and $\left\|\left.\phi_{n}(s)\right|_{s F}\right\| \geq 1-\epsilon$. It follows that

$$
\begin{aligned}
\left\|\phi_{n}(s)-\phi_{n}(e)\right\|= & \sum_{g \in G}\left|\phi_{n}(s)(g)-\phi_{n}(e)(g)\right| \\
= & \sum_{g \in F}\left|\phi_{n}(e)(g)-\phi_{n}(s)(g)\right|+\sum_{g \in s F}\left|\phi_{n}(e)(g)-\phi_{n}(s)(g)\right| \\
& \quad+\sum_{g \notin F \cup s F}\left|\phi_{n}(e)(g)-\phi_{n}(s)(g)\right| \\
\geq & 1-2 \epsilon+1-2 \epsilon+0 \\
= & 2-4 \epsilon .
\end{aligned}
$$

Hence $\left\|D \phi_{n}\right\|_{R=1} \geq 2$ for all $n$, and $D \phi \neq 0$. It follows that $\left[\mathbf{1}_{Q}\right] \notin \operatorname{Im} \pi_{*}$ in $H_{Q}^{0}(G, \mathbb{C})$ so by Theorem 7.3, $G$ does not admit an equivariant asymptotically invariant mean.

Theorem 10.4 Let $G$ be a countable residually finite group equipped with a left invariant metric $d$, and let $X=\bigsqcup_{\lambda \in \Lambda} X_{\lambda}$ be a countable box space for $(G, d)$ with the following properties:

(1) For every $g$ in $G$ there exists $\lambda \in \Lambda$ such that $d(e, g)=d\left(e_{\lambda}, g_{\lambda}\right)$.

(2) For each $\lambda \in \Lambda$ and for each $x \in X_{\lambda}$ there are finitely many elements $g \in G$ such that $d\left(e_{\lambda}, x\right)=d(e, g)$ and $g_{\lambda}=x$.

Then $G$ is metrically amenable if and only if $X$ has property $A$.

If $G$ is a finitely generated residually finite group, it admits a proper left invariant metric and any box space of $G$ will then satisfy conditions (1) and (2) above. Hence we recover Guentner's theorem. The disjoint union of the finite quotients $X=\bigsqcup_{n} \bigoplus_{i=1}^{n} \mathbb{Z}_{2}$ is a box space of the group $G=\bigoplus_{i \in \mathbb{N}} \mathbb{Z}_{2}$ which also satisfies conditions (1) and (2). (The second condition follows from the fact that while the metric is not proper, given any element $g \in G$ there are only finitely many geodesics in $G$ from $e$ to $g$.) Since $X$ is a union of finite cubes of all dimensions we recover Nowak's theorem since we have noted that $G$ is not metrically amenable.

Proof of Theorem 10.4 The proof that if $G$ is metrically amenable then $X$ has property A is identical to that given in our proof of Theorem 10.2, since that part of the proof did not invoke properness of the metric.

For the converse, we identify $\mathcal{E}^{0,-1}\left(G, \ell^{1}(G)\right)$ with a subspace $\mathcal{F}$ of $\mathcal{E}^{0,-1}\left(X, \ell^{1}(X)\right)$, and show that $\mathcal{E}^{0,-1}\left(X, \ell^{1}(X)\right)$ retracts onto $\mathcal{F}$. We deduce that $H_{W}^{0}\left(G, \ell^{1}(G)\right)$ injects into $H_{W}^{0}\left(X, \ell^{1}(X)\right)$. This injection is consistent with the isomorphism $H_{W}^{0}(G, \mathbb{C}) \cong$ 
$H_{W}^{0}(X, \mathbb{C})$, and we conclude that there is an equivariant asymptotically invariant mean in $H_{W}^{0}\left(G, \ell^{1}(G)\right)$ if and only if there is an asymptotically invariant mean in $H_{W}^{0}\left(X, \ell^{1}(X)\right)$.

Now suppose that $X$ has property A. As in the proof of Theorem 10.2 we will pull back the asymptotically invariant mean on $X$ to an equivariant asymptotically invariant mean on $G$, taking care to ensure that the pullback is compatible with the summation map $\pi$. We will use the weak-* completion.

As above let $q_{*}$ denote the pushforward from $\mathcal{E}^{0,-1}\left(G, \ell^{1}(G)\right)$ to $\mathcal{E}^{0,-1}\left(X, \ell^{1}(X)\right)$. We note that if $\psi \in \mathcal{E}^{0,-1}\left(X, \ell^{1}(X)\right)$ is in the image of $q_{*}$ then:

(i) $\psi$ is equivariant,

(ii) for $x \in X_{\lambda}, \psi(x)$ is supported in $X_{\lambda}$,

(iii) For $\lambda<\mu$ we have $\psi\left(e_{\lambda}\right)=\left(q_{\lambda \mu}\right)_{*} \psi\left(e_{\mu}\right)$.

Let $\mathcal{F}$ denote the subspace of $\mathcal{E}^{0,-1}\left(X, \ell^{1}(X)\right)$ of cochains with these properties.

We will first prove that the image of $q_{*}$ is $\mathcal{F}$. Let $R$ be a nonnegative integer and suppose that $\psi \in \mathcal{F}$ and for each $x \in X, \psi(x)$ is supported in the ball of radius $R$ about $x$. For $g \in G$ let

$$
\phi_{R}(e)(g)= \begin{cases}\lim _{\mu} \psi\left(e_{\mu}\right)\left(g_{\mu}\right), & d(e, g)=R \\ 0, & \text { otherwise. }\end{cases}
$$

We extend equivariantly. We will show that the limit exists, that $\phi_{R} \in \mathcal{E}^{0,-1}\left(G, \ell^{1}(G)\right)$, and $\left(q_{*} \phi_{R}\right)\left(e_{\lambda}\right)(y)=\psi\left(e_{\lambda}\right)(y)$ for $y \in X_{\lambda}$ with $d\left(e_{\lambda}, y\right)=R$.

To show that the limit exists, choose $\lambda$ such that $d\left(e_{\lambda}, g_{\lambda}\right)=R$, which is possible by hypothesis (1) of the Theorem. By hypothesis (2) the set $\mathcal{S}$ of $k \in G$ such that $d(e, k)=R$ and $k_{\lambda}=g_{\lambda}$ is finite. If $\mu$ is sufficiently large, by residual finiteness of $G, q_{\mu}$ is injective on $\mathcal{S}$. Now for any $v>\mu$ we have $\psi\left(e_{\mu}\right)=\left(q_{\mu \nu}\right)_{*}\left(\psi\left(e_{\nu}\right)\right)$ so

$$
\psi\left(e_{\mu}\right)\left(g_{\mu}\right)=\sum_{\substack{y \in X_{v} \\ y_{\mu}=g_{\mu}}} \psi\left(e_{\nu}\right)(y) .
$$

As $y_{\mu}=g_{\mu}, d\left(e_{\nu}, y\right) \geq d\left(e_{\mu}, g_{\mu}\right) \geq d\left(e_{\lambda}, g_{\lambda}\right)=R$, hence the only non-zero terms are for $d\left(e_{v}, y\right)=R$. We have $y=k_{v}$ for some $k \in G$ with $d(e, k)=R$. Then $k_{\lambda}=y_{\lambda}=g_{\lambda}$ so $k \in \mathcal{S}$ and $k_{\mu}=y_{\mu}=g_{\mu}$, so $k=g$ as $q_{\mu}$ is injective on $\mathcal{S}$. Hence there is only one term in the sum and we have $\psi\left(e_{\mu}\right)\left(g_{\mu}\right)=\psi\left(e_{\nu}\right)\left(g_{v}\right)$. The net is therefore ultimately constant, so it converges. 
We now show that $\phi_{R}(e)$ is in $\ell^{1}(G)$. Let $F$ be a finite subset of $G$. Then

$$
\sum_{g \in F}\left|\phi_{R}(e)(g)\right|=\lim _{\mu} \sum_{g \in F}\left|\psi\left(e_{\mu}\right)\left(g_{\mu}\right)\right| .
$$

Choosing $\mu$ sufficiently large so that $q_{\mu}$ is injective on $F$ we see that

$$
\sum_{g \in F}\left|\phi_{R}(e)(g)\right| \leq\left\|\psi\left(e_{\mu}\right)\right\|_{\ell^{1}} \leq\|\psi\| .
$$

As this holds for all finite $F$ we deduce that $\phi_{R}(e)$ is in $\ell^{1}(G)$, and $\left\|\phi_{R}(e)\right\|_{\ell^{1}} \leq\|\psi\|$. So $\phi_{R} \in \mathcal{E}^{0,-1}\left(G, \ell^{1}(G)\right)$ as required.

For $y \in X_{\lambda}$ with $d\left(e_{\lambda}, y\right)=R$ we have

$$
\left(q_{*} \phi_{R}\right)\left(e_{\lambda}\right)(y)=\sum_{g \in G, g_{\lambda}=y} \phi_{R}(e)(g)=\sum_{g \in \mathcal{T}} \phi_{R}(e)(g)
$$

where $\mathcal{T}$ is the set of all $g \in G$ such that $d(e, g)=R$ and $g_{\lambda}=y$. As $\mathcal{T}$ is finite by hypothesis (2), we can choose $\mu$ sufficiently large that $\phi_{R}(e)(g)=\psi\left(e_{\mu}\right)\left(g_{\mu}\right)$ for all $g \in \mathcal{T}$ and so that $q_{\mu}$ is injective on $\mathcal{T}$.

We note that $q_{\mu}(\mathcal{T})$ is the set of $z \in X_{\mu}$ such that $d\left(e_{\mu}, z\right)=R$ and $z_{\lambda}=y$. Since $\psi\left(e_{\mu}\right)(z)$ vanishes if $d\left(e_{\mu}, z\right)>R$ we have

$$
\left(q_{*} \phi_{R}\right)\left(e_{\lambda}\right)(y)=\sum_{g \in \mathcal{T}} \psi\left(e_{\mu}\right)\left(g_{\mu}\right)=\sum_{\substack{z \in X \mu \\ z_{\lambda}=y}} \psi\left(e_{\mu}\right)(z)=\psi\left(e_{\lambda}\right)(y)
$$

as required.

It now follows that if $R$ is a positive integer, then $\psi-q_{*} \phi_{R}$ is supported on the ball of radius $R-1$, while if $R=0$ then $\psi=q_{*} \phi_{R}$. Hence, by induction on $R$ every $\psi \in \mathcal{F}$ is in the image of $q_{*}$.

For a $\theta \in \mathcal{E}^{1,-1}\left(X, \ell^{1}(X)\right)$ define

$$
\|\theta\|_{R}^{\text {loc }}=\sup \left\|\theta\left(x_{0}, x_{1}\right)\right\|
$$

where the supremum is taken over all $\left(x_{0}, x_{1}\right) \in \bigsqcup_{\lambda} X_{\lambda}^{2}$ such that $d\left(x_{0}, x_{1}\right) \leq R$. We now establish the following claim:

Claim There exists a retraction $r$ from $\mathcal{E}^{0,-1}\left(X, \ell^{1}(X)\right)$ onto $\mathcal{F}$, such that for $\theta \in$ $\mathcal{E}^{0,-1}\left(X, \ell^{1}(X)\right)$, if $\pi_{*}(\theta)=\mathbf{1}$ then $\pi_{*}(r \theta)=\mathbf{1}$, and $\|\operatorname{Dr} \theta\|_{R}^{\text {loc }} \leq\|D \theta\|_{R}$. 
To prove this we will need to extract limits from bounded nets indexed by $\Lambda$; to do so we choose an ultrafilter $\omega$ extending the natural filter on the directed system $\Lambda$.

Given $\theta \in \mathcal{E}^{0,-1}\left(X, \ell^{1}(X)\right)$ we set $\bar{\theta}\left(e_{\lambda}\right)(y)=\frac{1}{\left|X_{\lambda}\right|} \sum_{z \in X_{\lambda}} \theta(z)(z y)$, for each $y \in X_{\lambda}$.

We now set

$$
\psi\left(e_{\lambda}\right)(y)=\lim _{\mu \in \omega} \sum_{\substack{z \in X_{\mu} \\ z_{\lambda}=y}} \bar{\theta}\left(e_{\mu}\right)(z),
$$

for each $y \in X_{\lambda}$ and set it to 0 for all other $y \in X$. We extend this equivariantly to a map from $X$ to $\ell^{1}(X)$. By definition this satisfies conditions (i) and (ii). It also satisfies condition (iii) as

$$
\begin{aligned}
\sum_{\substack{z \in X_{\mu} \\
z_{\lambda}=y}} \psi\left(e_{\mu}\right)(z) & =\sum_{\substack{z \in X_{\mu} \\
z_{\lambda}=y}} \lim _{\nu \in \omega} \sum_{\substack{w \in X_{\nu} \\
w_{\mu}=z}} \bar{\theta}\left(e_{\nu}\right)(w) \\
& =\lim _{\nu \in \omega} \sum_{\substack{z \in X_{\mu} \\
z_{\lambda}=y}} \sum_{\substack{w \in X_{\nu} \\
w_{\mu}=z}} \bar{\theta}\left(e_{\nu}\right)(w) \\
& =\lim _{\nu \in \omega} \sum_{\substack{w \in X_{\nu} \\
w_{\lambda}=y}} \bar{\theta}\left(e_{\nu}\right)(w)=\psi\left(e_{\lambda}\right)(y) .
\end{aligned}
$$

It is straightforward to verify that $\psi$ is bounded and of controlled supports, so we obtain a map $r: \mathcal{E}^{0,-1}\left(X, \ell^{1}(X)\right) \rightarrow \mathcal{F}$ as required. If $\theta \in F$ then $\bar{\theta}=\theta$ by equivariance and $\psi=\bar{\theta}$ by condition (iii) so the map $r$ is a retraction onto $\mathcal{F}$.

We now compute $\pi_{*}(\psi)$.

$$
\begin{aligned}
\pi_{*}(\psi)\left(e_{\lambda}\right) & =\sum_{y \in X_{\lambda}} \lim _{\mu \in \omega} \sum_{\substack{z \in X_{\mu} \\
z_{\lambda}=y}} \bar{\theta}\left(e_{\mu}\right)(z)=\lim _{\mu \in \omega} \sum_{\substack { y \in X_{\lambda} \\
\begin{subarray}{c}{z \in X_{\mu} \\
z_{\lambda}=y{ y \in X _ { \lambda } \\
\begin{subarray} { c } { z \in X _ { \mu } \\
z _ { \lambda } = y } }\end{subarray}} \bar{\theta}\left(e_{\mu}\right)(z) \\
& =\lim _{\mu \in \omega} \sum_{z \in X_{\mu}} \bar{\theta}\left(e_{\mu}\right)(z)=\lim _{\mu \in \omega} \sum_{z \in X_{\mu}} \frac{1}{\left|X_{\mu}\right|} \sum_{w \in X_{\mu}} \theta(w)(w z) \\
& =\lim _{\mu \in \omega} \frac{1}{\left|X_{\mu}\right|} \sum_{w \in X_{\mu}} \sum_{z \in X_{\mu}} \theta(w)(w z)=\lim _{\mu \in \omega} \frac{1}{\left|X_{\mu}\right|} \sum_{w \in X_{\mu}} \pi_{*}(\theta)(w) .
\end{aligned}
$$

In particular $\pi_{*}(\psi)$ is constant, and if $\pi_{*}(\theta)$ is the constant function $\mathbf{1}$ then $\pi_{*}(\psi)=\mathbf{1}$ as well.

We now consider the norm $\|D \psi\|_{R}^{\text {loc }}$. 
By equivariance it suffices to consider $\left\|\psi(x)-\psi\left(e_{\lambda}\right)\right\|_{\ell^{1}}$ where $x \in X_{\lambda}$ and $d\left(e_{\lambda}, x\right) \leq$ $R$. Pick $g \in G$ such that $d(e, g)=d\left(e_{\lambda}, x\right)$ and $g_{\lambda}=x$.

$$
\begin{aligned}
\left\|\psi(x)-\psi\left(e_{\lambda}\right)\right\|_{\ell^{1}} & =\sum_{y \in X_{\lambda}}\left|\psi(x)(y)-\psi\left(e_{\lambda}\right)(y)\right| \\
& =\lim _{\mu} \sum_{y \in X_{\lambda}}\left|\sum_{\substack{z \in X_{\mu} \\
z_{\lambda}=y}} \bar{\theta}\left(g_{\mu}\right)(y)-\bar{\theta}\left(e_{\mu}\right)(z)\right| \\
& \leq \lim _{\mu} \sum_{y \in X_{\lambda}} \sum_{\substack{z \in X_{\mu} \\
z_{\lambda}=y}}\left|\bar{\theta}\left(g_{\mu}\right)(y)-\bar{\theta}\left(e_{\mu}\right)(z)\right| \\
& =\lim _{\mu} \sum_{z \in X_{\mu}}\left|\bar{\theta}\left(g_{\mu}\right)(y)-\bar{\theta}\left(e_{\mu}\right)(z)\right| \\
& \leq\|D \bar{\theta}\|_{R}^{\text {loc }} .
\end{aligned}
$$

It is easy to see that $\|D \bar{\theta}\|_{R}^{\text {loc }} \leq\|D \theta\|_{R}$ since taking the average is a norm-decreasing operation. Hence $\|D \psi\|_{R}^{\text {loc }} \leq\|D \theta\|_{R}$ as required.

We complete the proof of Theorem 10.4 as follows.

Note that for any $\phi \in \mathcal{E}^{0,-1}\left(G, \ell^{1}(G)\right)$ and for any pre-images $g_{0}, g_{1} \in G$ of $x_{0}, x_{1} \in$ $X_{\lambda}$,

$$
\begin{aligned}
D q_{*} \phi\left(x_{0}, x_{1}\right) & =x_{1}\left(q_{\lambda}\right)_{*} \phi(e)-x_{0}\left(q_{\lambda}\right)_{*} \phi(e) \\
& =\left(q_{\lambda}\right)_{*} \phi\left(g_{1}\right)-\left(q_{\lambda}\right)_{*} \phi\left(g_{0}\right) \\
& =\left(q_{\lambda}\right)_{*} D \phi\left(g_{0}, g_{1}\right) .
\end{aligned}
$$

Since there exist pre-images with $d\left(g_{0}, g_{1}\right)=d\left(x_{0}, x_{1}\right)$ we deduce that $\left\|D q_{*} \phi\right\|_{R}^{\text {loc }} \leq$ $\|D \phi\|_{R}$. In fact we have equality: for each $g_{0}, g_{1}$ in $G$, the norm $\left\|\left(q_{\lambda}\right)_{*} D \phi\left(g_{0}, g_{1}\right)\right\|$ converges to $\left\|D \phi\left(g_{0}, g_{1}\right)\right\|$ (by residual finiteness). By the same argument $\left\|q_{*} \phi\right\|=$ $\|\phi\|$ for all $\phi$, hence $q_{*}$ is injective.

Having noted that $q_{*}$ is an isometric bijection $\mathcal{E}^{0,-1}\left(G, \ell^{1}(G)\right) \rightarrow \mathcal{F}$ we obtain a bounded map $q_{*}^{-1} r: \mathcal{E}^{0,-1}\left(X, \ell^{1}(X)\right) \rightarrow \mathcal{E}^{0,-1}\left(G, \ell^{1}(G)\right)$. This extends to a map $\mathcal{E}_{W}^{0,-1}\left(X, \ell^{1}(X)\right) \rightarrow \mathcal{E}_{W}^{0,-1}\left(G, \ell^{1}(G)\right)$ and for any invariant mean $\mu \in \mathcal{E}_{W}^{0,-1}\left(X, \ell^{1}(X)\right)$ we obtain an element $\mu^{\prime} \in \mathcal{E}_{W}^{0,-1}\left(G, \ell^{1}(G)\right)$. We will show that $\mu^{\prime}$ is an equivariant, asymptotically invariant mean for $G$, which will establish that $G$ is metrically amenable. Since $\pi_{*}(\mu)=\mathbf{1}_{W}, \pi_{*}\left(\mu^{\prime}\right)=\mathbf{1}_{W}$, it only remains to show that $D \mu^{\prime}=0$.

To do this we use $q_{*}^{-1} r$ to induce a map from $D \mathcal{E}^{0,-1}\left(X, \ell^{1}(X)\right)$ to $D \mathcal{E}^{0,-1}\left(G, \ell^{1}(G)\right)$, which, abusing notation, we will also denote by $q_{*}^{-1} r$. Define $q_{*}^{-1} r(D \theta)=D\left(q_{*}^{-1} r(\theta)\right)$; 
this is well defined as $D$ is injective on $\mathcal{E}^{0,-1}\left(X, \ell^{1}(X)\right)$. The map $q_{*}^{-1} r$ is continuous since for $\theta \in \mathcal{E}^{0,-1}\left(X, \ell^{1}(X)\right)$ and $\phi=q_{*}^{-1} r(\theta)$ we have

$$
\left\|q_{*}^{-1} r(D \theta)\right\|_{R}=\|D \phi\|_{R}=\left\|D q_{*} \phi\right\|_{R}^{\text {loc }}=\|\operatorname{Dr} \theta\|_{R}^{\text {loc }} \leq\|D \theta\|_{R} .
$$

Now the weak-* completion of $D \mathcal{E}^{0,-1}\left(X, \ell^{1}(X)\right)$ injects into the weak-* completion of $\mathcal{E}^{1,-1}\left(X, \ell^{1}(X)\right)$ so by continuity $q_{*}^{-1} r$ extends and in particular $D \mu^{\prime}=$ $q_{*}^{-1} r D \mu=0$. Hence an asymptotically invariant mean for $X$ maps to an equivariant asymptotically invariant mean for $G$.

\section{References}

[1] J Brodzki, G A Niblo, P W Nowak, N J Wright, Amenable actions, invariant means and bounded cohomology arXiv:1004.0295

[2] J Brodzki, G A Niblo, P W Nowak, N J Wright, A homological characterization of topological amenability arXiv:1008.4154

[3] J Brodzki, G A Niblo, NJ Wright, Pairings, duality, amenability, and bounded cohomology, to appear in J. Eur. Math. Soc. (JEMS) arXiv:1003.2584

[4] R G Douglas, P W Nowak, Invariant expectations and vanishing of bounded cohomology for exact groups, J. Topol. Anal. 3 (2011) 89-107 MR2784765

[5] S M Gersten, Cohomological lower bounds for isoperimetric functions on groups, Topology 37 (1998) 1031-1072 MR1650363

[6] N Higson, J Roe, Amenable group actions and the Novikov conjecture, J. Reine Angew. Math. 519 (2000) 143-153 MR1739727

[7] B E Johnson, Cohomology of Banach Algebras, Mem. Amer. Math. Soc. 127, American Mathematical Society, Providence, RI (1972)

[8] A Khukhro, N J Wright, Expanders and property A, Algebr. Geom. Topol. 12 (2012) $37-47$

[9] P W Nowak, Coarsely embeddable metric spaces without Property A, J. Funct. Anal. 252 (2007) 126-136 MR2357352

[10] N Ozawa, Amenable actions and exactness for discrete groups, C. R. Acad. Sci. Paris Sér. I Math. 330 (2000) 691-695 MR1763912

[11] H Reiter, Classical harmonic analysis and locally compact groups, Clarendon Press, Oxford (1968) MR0306811

[12] J Roe, Exotic cohomology and index theory, Bull. Amer. Math. Soc. 23 (1990) 447-453 MR1049433

[13] J Roe, Lectures on coarse geometry, University Lecture Series 31, American Mathematical Society (2003) MR2007488 
[14] J von Neumann, Zur allgemeinen Theorie des Maßes, Fund. Math. 13 (1929) 73-111

[15] G Yu, The coarse Baum-Connes conjecture for spaces which admit a uniform embedding into Hilbert space, Invent. Math. 139 (2000) 201-240 MR1728880

School of Mathematics, University of Southampton,

Highfield, Southampton, SO17 1SH, England

J.Brodzki@soton.ac.uk, G.A.Niblo@soton.ac.uk, N.J.Wright@soton.ac.uk

Proposed: Danny Calegari

Received: 27 May 2011

Seconded: Peter Teichner, Steve Ferry Accepted: 11 November 2011 